\title{
32. PELAGIC AND HEMIPELAGIC SEDIMENTS OF THE IZU-BONIN REGION, LEG 126: GEOCHEMICAL AND COMPOSITIONAL FEATURES ${ }^{1}$
}

\author{
Akira Nishimura, ${ }^{2}$ Naoki Mita, ${ }^{3}$ and Masato Nohara ${ }^{2}$
}

\begin{abstract}
Chemical analyses were performed on major, minor, and rare-earth elements of pelagic and hemipelagic sediments of the forearc, arc, and backarc sites of the Izu-Bonin Arc, Ocean Drilling Program Leg 126. Analyses of the hemipelagic and pelagic sediments of this area indicate that the chemical composition of this arc is highly affected by the chemical composition of rocks of this arc as a source of sediments. The Oligocene sediments, which are characterized by high $\mathrm{MgO}$ contents, reflect the chemical composition of the Paleogene volcanic rocks of the immature arc. Moreover, the late Miocene to Quaternary sediments with low $\mathrm{MgO}$ contents are attributed to the composition of the present arc. We also suggest that the sedimentation rates and topography of the sedimentary basin affect the $\mathrm{MnO}$ and $\mathrm{SiO}_{2}$ contents of pelagic and hemipelagic sediments.
\end{abstract}

\section{INTRODUCTION}

The Izu-Bonin Arc is an intraoceanic arc between the northwestern Pacific and the Philippine Sea that was formed from volcanic activities, backarc rifting, and spreading processes since Eocene time (Honza and Tamaki, 1985). Ocean Drilling Program (ODP) Leg 126 is the first deep-sea project to drill in the Izu-Bonin Arc area that transects the entire arc (Fig. 1).

The geochemistry of ocean-floor pelagic sediments has been studied to solve various problems, that is, the major source of sediments in the area, the geologic history of hydrothermal activity, the origin of manganese nodules, and so on (Leinen, 1987; Sugisaki, $1980 \mathrm{~b}$; Nohara and Kato, 1985). However, the same kinds of studies on near-land areas are very few. The chemical compositions of argillaceous sediments near the arc were studied on sediments from the Japan Trench area (Sugisaki, 1980a; Nohara, 1980). The chemical compositions of argillaceous sediments from the Izu-Bonin Arc were reported on only the surface sediments obtained by a piston and a gravity cores (Sugisaki and Kinoshita, 1981). The objectives of this paper are to determine the geologic changes in the chemical features of the sediments and to clarify the origins of finer grained sediments of the forearc and backarc of the Izu-Bonin Arc, which are related to the geologic history of the arc. Furthermore, attention was concentrated on the affect of hydrothermal activity on the composition of sediments in the Sumisu Rift, which is an active rift that is expected to contain hydrothermal circulation (Yamazaki, 1988; Nakao et al., 1990). To accomplish these objectives, the bulk chemical composition of 103 samples and the rare-earth elements of 13 samples from Leg 126 were determined and examined.

\section{ANALYTICAL METHODS}

The geochemical data of pelagic and hemipelagic sediments are compared with the lithologic data. The lithologic data are mainly based on core descriptions from the Proceedings of the Ocean Drilling Program, Initial Reports for Leg 126 (Taylor, Fujioka, et al., 1990). Authigenic components in the sediments are sometimes related

\footnotetext{
${ }^{1}$ Taylor, B., Fujioka, K., et al., 1992. Proc. ODP, Sci. Results, 126: College Station, TX (Ocean Drilling Program).

${ }^{2}$ Marine Geology Department, Geological Survey of Japan, Higashi 1-1-3, Tsukuba, Ibaraki 305, Japan.

${ }^{3}$ Geochemistry Department, Geological Survey of Japan, Higashi 1-1-3, Tsukuba, Ibaraki 305, Japan.
}

to the sedimentation rate. Sedimentation rates were recalculated for muddy sediments, excluding such instantaneous deposits as volcanic ashes, turbidites, and debris-flow deposits, based on the original on-board visual core descriptions and formation microscanner (FMS) columns (Hiscott et al., this volume).

\section{Determination of Major and Minor Chemical Elements}

Sediment samples including seawater were dried at room temperature and ground well. The moisture contents $\left(\mathrm{H}_{2} \mathrm{O}\right)$ of samples were determined after drying for $3 \mathrm{hr}$ at $110^{\circ} \mathrm{C}$.

Total carbon and sulfur contents were measured by infrared spectrometric determination and high-frequency furnace. Calcium carbonate contents were converted from nonorganic carbon contents and were measured by the same methods above after precombustion at $450^{\circ} \mathrm{C}$ for $12 \mathrm{hr}$. Organic carbon contents were calculated by subtracting the nonorganic carbon contents from the total for carbon contents.

Dissolution of sediment samples was achieved to use $\mathrm{HCl}(35 \%)$ and $\mathrm{HF}(49 \%) . \mathrm{SiO}_{2}, \mathrm{Al}_{2} \mathrm{O}_{3}$, and $\mathrm{TiO}_{2}$ were measured by atomic absorption spectrometry with $\mathrm{N}_{2} \mathrm{O}-\mathrm{C}_{2} \mathrm{H}_{2}$ flame. Other elements were measured by atomic absorption spectrometry with air- $\mathrm{C}_{2} \mathrm{H}_{2}$ flame. All analytical values were converted to $110^{\circ} \mathrm{C}$ dried basis, excluding the moisture contents. Total $\mathrm{Fe}$ contents were calculated as $\mathrm{Fe}_{2} \mathrm{O}_{3}$, and $\mathrm{CaO}$ contents were calculated by subtracting the $\mathrm{CaO}$ contents as calcium carbonate from the total $\mathrm{CaO}$.

\section{Determination of Strontium and Rare-earth Elements}

For $\mathrm{Sr}$ analysis, the sediment samples were washed with deionized water to remove the effect of seawater. Afterward, the samples were repeatedly washed with super-grade alcohol. Because the analyzed sediment samples are mainly composed of calcium carbonate, the dried sample was dissolved in $2 \mathrm{~N} \mathrm{HCl}$ and immediately transfered to the ion-exchange column to minimize the effect of silica minerals. The $\mathrm{Sr}$ isotopic ratio was measured by MAT 262 with multicollector system. Isotopic data are fractionation corrected to ${ }^{87} \mathrm{Sr} /{ }^{86} \mathrm{Sr}=0.1194$. Mean measured standard values are ${ }^{87} \mathrm{Sr} /{ }^{86} \mathrm{Sr}=0.710223$ for NBS987 with a total range of \pm 0.000010 .

For rare-earth-element (REE) analyses, the sediment samples were washed with deionized water and were dissolved in mixtures of ultra-pure $\mathrm{HClO}_{4}, \mathrm{HCl}$, and $\mathrm{HF}$. The REEs were determined by ICP-MS, after removing major elements by ion-exchange resin. The precision of the replicate analyses is better than $5 \%$. 


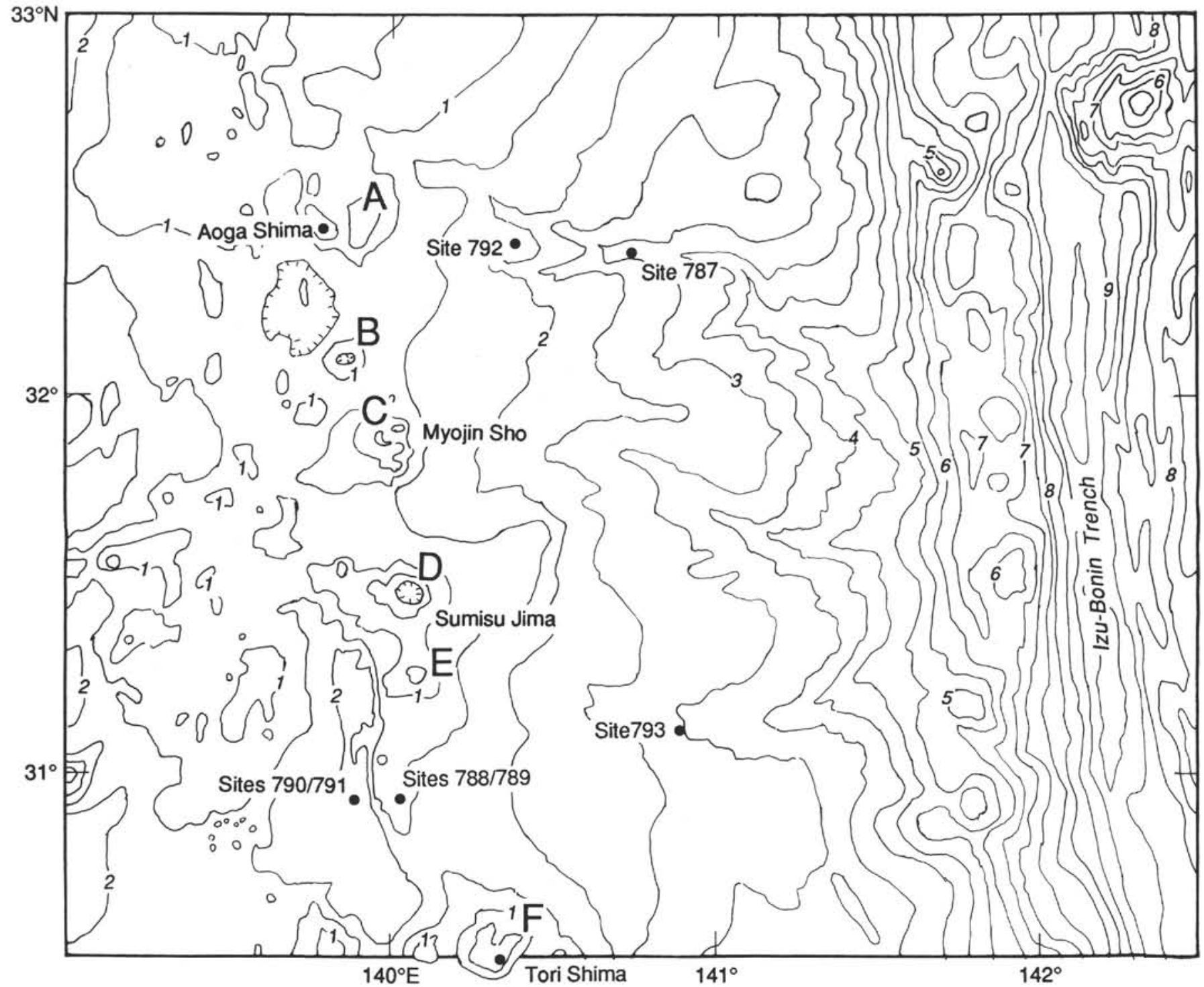

Figure 1. Bathymetric map showing sites drilled on Leg 126 and volcanoes in the vicinity. Bathymetric map of Izu-Bonin is based on Taylor et al. (1990). Contour interval in 500-m intervals. A = Higashi-Aoga Shima Caldera, B = Kita-Beyonesu Caldera, C = Myojin-sho Caldera, D = Sumisu Jima Caldera, E = Minami-Sumisu Jima Caldera, and $\mathrm{F}=$ Tori Shima Caldera.

\section{GEOLOGIC SETTING AND SAMPLES}

The Izu-Bonin Arc is an active intraoceanic arc between the northwestern Pacific and the Philippine Sea that has been forming since the Eocene by intense volcanisms (Honza and Tamaki, 1985). There are submarine calderas in the northern part of the Izu-Bonin Arc, where Leg 126 was drilled (Fig. 1). Backarc rifts are discontinuously present along the volcanic front. The Sumisu Rift, which was also drilled on Leg 126, is a backarc rift just behind the volcanic front between Sumisu Jima and Tori Shima islands (Fig. 1) (Honza and Tamaki, 1985; Brown and Taylor, 1988; Murakami, 1988; Taylor et al., 1990). The forearc basin, which was drilled on Leg 126, was formed in the early Oligocene between the frontal- and outer-arc highs of the Paleogene volcanic basement (Taylor, Fujioka, et al., 1990).

\section{Forearc Sites}

Three sites $(787,792$, and 793) drilled on Leg 126 are composed of geologic sections from the early Oligocene to the Quaternary. The age-depth curves of the three sites are similar to each other (Shipboard
Scientific Party, 1990a), which suggests that a similar geologic history dominated throughout the forearc basin. The lower to middle parts of the Oligocene sequences consist of sediment gravity flow deposits with a high sedimentation rate, such as turbidites and debris-flow deposits. The latest Oligocene and lower Miocene sequences are dominantly of pelagic mudstone with a very low sedimentation rate. The upper Miocene to Quaternary sequence consists of hemipelagite with a high content of volcanogenic materials.

\section{Arc Site}

The Site 788 sequence drilled on the eastern flank top of the Sumisu Rift is mainly composed of pumice deposits. Pelagic claystones/ siltstones were found in the lower part of this sequence (Subunits IIA and IIB).

\section{Backarc Sites}

The sedimentary sequences in the backarc sites are Quaternary aged $(0-1.1 \mathrm{Ma})$ and are composed of two units. The two units contrast 
highly in the contents of their ash and tephra layers, but hemipelagites in the two units possess a similar lithology in their high calcareous nannofossils contents. Two sites that are $2.4 \mathrm{~km}$ apart show the same lithologic units with different thicknesses (Shipboard Scientific Party, 1990b).

Hemipelagic and pelagic sediments were selected for chemical analyses from almost all the units of all Leg 126 sites on the Izu-Bonin Arc (Fig. 1). The chemical analyses of the major and minor elements were performed on 103 samples. The samples were checked visually and selected as hemipelagic and pelagic sediments. The analyses of REEs were performed on 13 selected samples of backarc Site 791 and forearc Site 792, which are representative of almost all the lithologic units of Leg 126. Chemical analyses were performed on almost all the units of the sedimentary sequences of all sites; however, for Site 788 only one sample of Subunit IIA was available for analysis because most of the sequences in this site are composed of volcanogenic sediments.

\section{GEOCHEMICAL FEATURES OF MAJOR AND MINOR ELEMENTS}

The results of the chemical analyses are listed in Table 1. All major components are given in weight percent of oxides, and minor ones in weight percent of atoms. Furthermore, to determine the origin of sediments excluding biogenic effects (discussed later), the contents of the major elements were recalculated on the basis of being carbonate free (Tables 2 and 3). The vertical changes in chemical composition are shown in Figures 2 through 6. Moreover, the $\mathrm{SiO}_{2}-\mathrm{Al}_{2} \mathrm{O}_{3}-10 \mathrm{TiO}$ and $\mathrm{Fe}_{2} \mathrm{O}_{3}-\mathrm{MgO}-\mathrm{TiO}_{2}$ triangles (Sugisaki, 1978) show the features of the sediments (Figs. 7 and 8). The elements used in these triangles are resistants for chemical weathering.

Throughout the core sequences of all the sites, the calcium carbonate content ranges from $1 \%$ to $46 \%$, and the carbonate-free contents of the major elements range as follows: $\mathrm{SiO}_{2}, 44 \%-68 \% ; \mathrm{Al}_{2} \mathrm{O}_{3}, 7 \%-21 \%$; $\mathrm{Fe}_{2} \mathrm{O}_{3}, 3 \%-11 \% ; \mathrm{TiO}_{2}, 0.1 \%-0.9 \% ; \mathrm{CaO}, 1 \%-26 \% ; \mathrm{MgO}, 0 \%-8 \%$; $\mathrm{Na}_{2} \mathrm{O}, 1 \%-5 \% ; \mathrm{K}_{2} \mathrm{O}, 0.4 \%-4.1 \%$; and $\mathrm{MnO}, 0.1 \%-1.4 \%$. The contents of rare metals range as follows: $\mathrm{Co}, 6-160 \mathrm{ppm} ; \mathrm{Ni}, 0-50 \mathrm{ppm} ; \mathrm{Cu}$, 0-200 ppm; Zn, 40-2370 ppm; Pb, 0-250 ppm; Li, 0-50 ppm; and Cr, 4-110 ppm. The contents of $\mathrm{Cu}$ and $\mathrm{Ni}$ in the sediments are very low compared with Pacific deep-sea sediments and Philippine Sea sediments (Mita et al., 1982; Sugisaki, 1980b).

\section{Forearc Sites}

The sequences of forearc Sites 787, 792, and 793 show the vertical changes of chemical composition of the sediments since the Oligocene age (Tables 1-2 and Figs. 2-4). The sequences of early to late Oligocene age show high $\mathrm{Fe}_{2} \mathrm{O}_{3}$ and $\mathrm{MgO}$ contents. The sequences of the Pliocene to the Quaternary show lower $\mathrm{SiO}_{2}$ and higher $\mathrm{Fe}_{2} \mathrm{O}_{3}$ contents compared with the backarc Quaternary sequences. The $\mathrm{CaO}$ content of Site 792 is lowest in the upper part of Unit IV (late Oligocene) and that of Site 793 is lowest in Unit IV (early Miocene).

\section{Arc Sites}

One sample from Subunit IIA has a similar chemical composition to that of Unit I of forearc Site 792. However, its contents of MgO, $\mathrm{CaO}$, and $\mathrm{MnO}$ are slightly higher than those of a later unit (Table 2).

\section{Backarc Sites}

The sequences of the two backarc Sites 790 and 791 consist of two lithologic units. Their chemical compositions are similar to each other, and they show higher mean contents of $\mathrm{SiO}_{2}$ compared with those of the forearc sites. The $\mathrm{CaO}$ and $\mathrm{MgO}$ contents of Unit II are higher than those of Unit I at backarc Sites 790 and 791 (Tables 2-3 and Figs. 5-6).

\section{GEOCHEMICAL CHARACTERISTICS OF REES AND STRONTIUM}

The results obtained from the REE analyses for Leg 126 are given in Table 4, and the corresponding chondrite-normalized REE patterns are shown in Figures 9 and 10. The REE abundances of the sediments from Sites 791 and 792 are generally higher than those of oceanic basalts. Their general shapes show that all sediments are enriched in light REEs and have a marked discontinuity in heavy REEs. Enrichment in light REEs is more marked at Site 791 than at Site 792 (Figs. 9 and 10 ).

The $\mathrm{Sr}$ isotopic ratios of marine carbonate minerals are assumed to be identical to those of seawater at the time of deposition, provided that they have not been altered during diagenesis, dolomitization, or regional metamorphism. The ${ }^{87} \mathrm{Sr} /{ }^{86} \mathrm{Sr}$ ratios in the ocean have changed systematically during Phanerozoic time (Peterman et al., 1970). Burke et al. (1982) obtained the average $\mathrm{Sr}$ isotopic ratio, $0.70910 \pm 0.00004$, by analyzing 42 samples of modern marine carbonate from all over the world. The $\mathrm{Sr}$ isotopic ratios of carbonates from the pelagic carbonate sediments of Unit III of Site 792 are $0.708129 \pm 0.000016$ (Sample 126792E-28R-1, 57-59 cm) and 0.708237 \pm 0.000012 (Sample 126$792 \mathrm{E}-31 \mathrm{R}-1,31-32 \mathrm{~cm}$ ), which are distinctly different from those of modern marine carbonates.

\section{MAJOR SOURCE OF PELAGIC AND HEMIPELAGIC SEDIMENTS}

\section{Biogenic Components in Chemical Composition}

Smear slide observations of the hemipelagic and pelagic sediments suggest that the major biogenic components of this area are

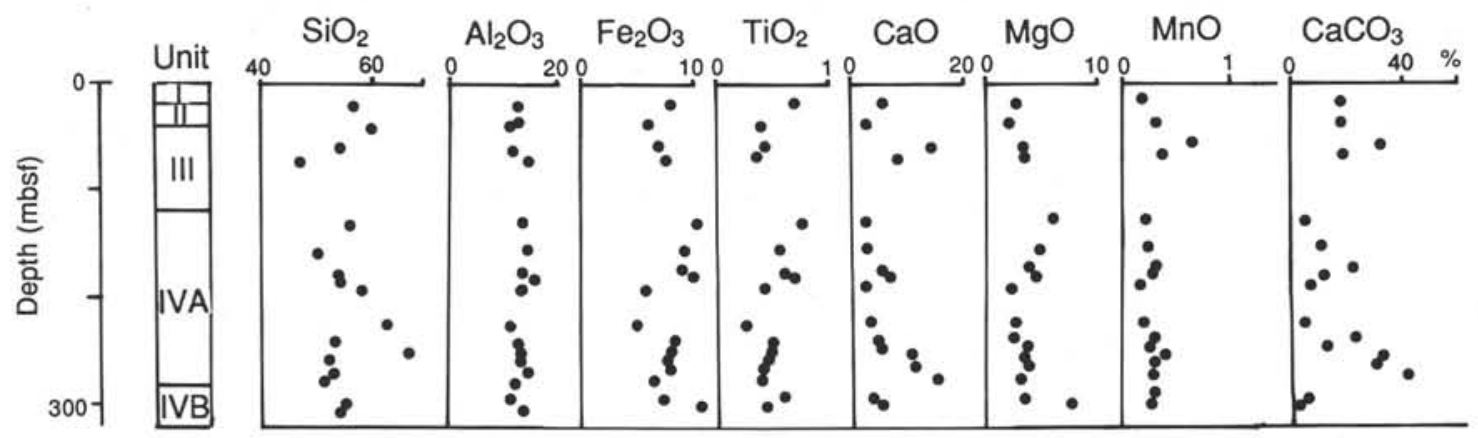

Figure 2. Vertical change of carbonate-free chemical compositions and carbonate content, Site 787. 
Table 1. Chemical compositions of pelagic and hemipelagic sediments of Leg 126, Izu-Bonin Arc.

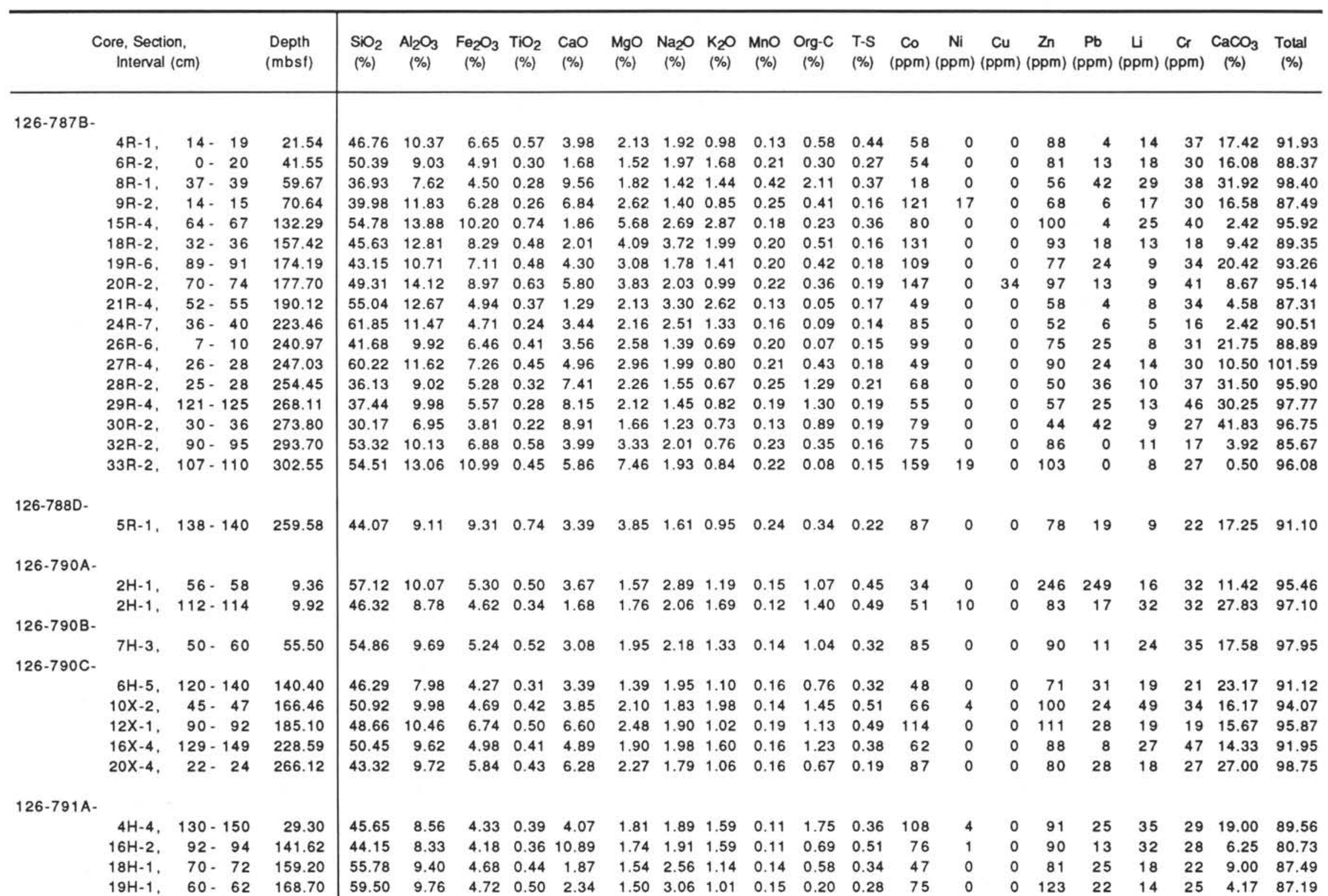


Table 1 (continued).

\begin{tabular}{llll|llllllllllllllllllllll}
$22 \mathrm{H}-6$, & $45-$ & 65 & 204.85 & 55.21 & 9.97 & 4.38 & 0.43 & 1.96 & 1.62 & 2.59 & 1.24 & 0.18 & 0.42 & 0.22 & 70 & 0 & 0 & 88 & 14 & 24 & 4 & 10.33 & 88.56
\end{tabular} $\begin{array}{lllllllllllllllllllllllllll}39 X-1, & 23- & 25 & 352.53 & 53.74 & 9.28 & 4.33 & 0.50 & 3.35 & 1.35 & 2.44 & 1.99 & 0.15 & 0.89 & 0.37 & 56 & 0 & 0 & 76 & 0 & 28 & 26 & 7.17 & 85.57\end{array}$ 126-791B-

13R-1, $\quad 97-99 \quad 503.07$ 22R-1, $\quad 21$ - $22 \quad 589.21$ 23R-1, $125-126 \quad 599.95$ 23R-4, $6-7 \quad 603.26$ 24R-1, $62-64$ 609.02 25R-2, $\quad 51$ - $52 \quad 620.11$ 25R-2, $\quad 64-66 \quad 620.24$ $27 R-1, \quad 30-31 \quad 637.30$ 31R-3, $144-146 \quad 680.04$ $34 \mathrm{R}-2, \quad 139-141 \quad 707.49$ $38 \mathrm{R}-3, \quad 110-112 \quad 745.35$ 39R-2, $\quad 45-46 \quad 754.35$ 40R-3, $\quad 10-11 \quad 765.20$ 41R-3, 77 - $79 \quad 775.47$ $42 \mathrm{R}-2, \quad 72-73 \quad 783.52$ 43R-1, $31-32 \quad 791.31$ 43R-1, $136-137 \quad 792.36$ $\begin{array}{llll}46 \mathrm{R}-2 & 34-36 \quad 821.84\end{array}$ $\begin{array}{lllllllllllll}45.58 & 8.51 & 3.92 & 0.37 & 3.98 & 1.59 & 1.71 & 1.69 & 0.17 & 1.32 & 0.46 & 80 & 10\end{array}$ $\begin{array}{lllllllllll}47.48 & 7.86 & 3.34 & 0.31 & 4.90 & 1.71 & 1.60 & 1.68 & 0.15 & 1.93 & 0.66\end{array}$ $\begin{array}{llllllllllll}39.68 & 7.05 & 3.08 & 0.27 & 4.88 & 1.19 & 1.46 & 1.53 & 0.09 & 2.00 & 0.47 & 36\end{array}$ $\begin{array}{llllllllllll}50.03 & 5.76 & 2.42 & 0.23 & 5.13 & 1.22 & 1.41 & 1.35 & 0.13 & 2.20 & 0.89 & 17\end{array}$ $\begin{array}{lllllllllllll}51.79 & 10.21 & 5.58 & 0.45 & 3.74 & 2.27 & 2.04 & 1.25 & 0.16 & 0.59 & 0.32 & 112\end{array}$ $\begin{array}{llllllllllll}49.30 & 11.01 & 7.49 & 0.58 & 5.83 & 2.89 & 1.99 & 0.76 & 0.19 & 0.63 & 0.48 & 103\end{array}$ $\begin{array}{llllllllllll}40.20 & 8.47 & 4.57 & 0.38 & 2.86 & 1.79 & 1.69 & 1.16 & 0.18 & 0.66 & 0.23 & 114\end{array}$ $\begin{array}{llllllllllll}48.34 & 8.01 & 3.30 & 0.34 & 0.72 & 0.94 & 2.21 & 1.12 & 0.16 & 0.40 & 0.29 & 61\end{array}$ $\begin{array}{llllllllllll}31.20 & 5.57 & 2.54 & 0.17 & 5.71 & 1.16 & 1.38 & 1.29 & 0.14 & 0.92 & 0.32 & 6\end{array}$ $\begin{array}{lllllllllllll}44.51 & 6.77 & 2.53 & 0.19 & 2.13 & 1.03 & 1.83 & 1.48 & 0.13 & 0.78 & 0.26 & 52\end{array}$ $\begin{array}{llllllllllll}50.15 & 9.34 & 6.43 & 0.51 & 4.71 & 2.38 & 1.96 & 1.37 & 0.19 & 0.97 & 0.21 & 134\end{array}$ $\begin{array}{llllllllllll}43.22 & 9.31 & 6.04 & 0.50 & 8.43 & 2.87 & 1.72 & 1.18 & 0.14 & 1.14 & 1.81 & 115\end{array}$ $\begin{array}{lllllllllllll}31.37 & 6.11 & 3.37 & 0.29 & 7.84 & 1.53 & 1.49 & 1.03 & 0.18 & 0.72 & 0.31 & 109\end{array}$ $\begin{array}{llllllllllll}28.03 & 5.53 & 3.38 & 0.20 & 14.10 & 1.39 & 1.25 & 1.02 & 0.13 & 0.74 & 0.38 & 69\end{array}$ $\begin{array}{lllllllllllll}40.85 & 8.15 & 4.27 & 0.38 & 4.74 & 1.95 & 1.61 & 1.46 & 0.12 & 0.65 & 0.49 & 91\end{array}$ $\begin{array}{llllllllllll}48.05 & 6.05 & 3.76 & 0.29 & 0.78 & 0.00 & 1.28 & 2.11 & 0.10 & 0.70 & 3.63 & 32\end{array}$ $\begin{array}{lllllllllllll}36.47 & 6.96 & 3.88 & 0.29 & 2.88 & 1.83 & 1.46 & 1.17 & 0.26 & 0.21 & 0.33 & 54\end{array}$ $\begin{array}{lllllllllllll}36.53 & 6.56 & 3.30 & 0.20 & 3.31 & 1.30 & 1.59 & 1.06 & 0.14 & 0.26 & 0.60 & 79\end{array}$

126-792A-

$2 \mathrm{H}-3, \quad 140-142 \quad 14.10$ $3 \mathrm{H}-6, \quad 72-92 \quad 27.32$ $4 \mathrm{H}-6, \quad 100-102 \quad 36.90$ $9 \mathrm{H}-2, \quad 19-21 \quad 77.01$

126-792B-

$8 \mathrm{X}-1, \quad 112-114 \quad 109.02$ $9 \mathrm{X}-2, \quad 13-15 \quad 119.13$ 126-792E-

1R-1, 17 - $19 \quad 135.77$ $\begin{array}{llll}2 R-1, & 7-9 & 9 & 145.27\end{array}$ $18 \mathrm{R}-1, \quad 70-72 \quad 300.20$ 19R-2, $120-122 \quad 311.70$ $21 \mathrm{R}-1, \quad 34-35 \quad 328.64$ 27R-3, $100-102 \quad 389.42$ $30 R-4, \quad 30-50 \quad 420.00$ $31 \mathrm{R}-1, \quad 57$ - $59 \quad 425.47$ 36R-4, $\quad 2$ - $5 \quad 477.72$ $37 R-2, \quad 62-63 \quad 484.92$ 38R-2, $\quad 84-87 \quad 494.84$ 39R-3, $\quad 1$ - 3 505.21 40R-5, $\quad 85-87 \quad 518.75$ $\begin{array}{llllllllllll}54.52 & 15.71 & 7.85 & 0.64 & 5.15 & 2.44 & 2.42 & 0.81 & 0.18 & 0.24 & 0.22 & 124\end{array}$ $\begin{array}{lllllllllllll}45.77 & 14.63 & 7.55 & 0.50 & 6.61 & 2.88 & 1.89 & 0.85 & 0.15 & 0.60 & 0.32 & 106\end{array}$ $\begin{array}{llllllllllll}43.95 & 11.71 & 6.22 & 0.46 & 4.69 & 1.95 & 1.94 & 0.93 & 0.13 & 0.66 & 0.26 & 121\end{array}$ $\begin{array}{llllllllllll}39.78 & 8.37 & 4.98 & 0.36 & 5.40 & 1.57 & 1.97 & 0.93 & 0.12 & 0.68 & 0.21 & 70\end{array}$

$\begin{array}{llllllllllll}49.86 & 14.11 & 8.79 & 0.65 & 6.43 & 2.55 & 2.16 & 0.66 & 0.19 & 0.41 & 0.29 & 154\end{array}$ $\begin{array}{lllllllllllll}51.28 & 10.73 & 4.17 & 0.29 & 3.74 & 1.23 & 2.41 & 1.18 & 0.13 & 0.60 & 0.29 & 69\end{array}$

$\begin{array}{llllllllllll}45.88 & 12.05 & 8.11 & 0.58 & 5.75 & 2.61 & 2.01 & 0.71 & 0.17 & 0.50 & 0.23 & 145\end{array}$ $\begin{array}{lllllllllllll}47.42 & 13.30 & 7.81 & 0.54 & 6.42 & 2.46 & 2.07 & 0.74 & 0.18 & 0.41 & 0.32 & 152\end{array}$ $\begin{array}{llllllllllll}47.14 & 12.06 & 7.66 & 0.48 & 4.78 & 2.55 & 2.17 & 0.95 & 0.18 & 0.22 & 0.16 & 108\end{array}$ $\begin{array}{llllllllllll}42.92 & 10.67 & 6.93 & 0.40 & 3.98 & 2.25 & 1.82 & 0.74 & 0.25 & 0.08 & 0.20 & 118\end{array}$ $\begin{array}{llllllllllll}55.02 & 14.55 & 6.75 & 0.41 & 4.83 & 2.01 & 2.11 & 0.84 & 0.21 & 0.12 & 0.18 & 135\end{array}$ $\begin{array}{llllllllllll}30.36 & 6.23 & 2.90 & 0.06 & 2.75 & 1.47 & 1.26 & 1.06 & 0.22 & 0.59 & 0.28 & 8\end{array}$ $\begin{array}{llllllllllll}30.44 & 7.48 & 4.37 & 0.25 & 3.29 & 2.17 & 1.11 & 2.53 & 0.41 & 0.64 & 0.26 & 45\end{array}$ $\begin{array}{llllllllllll}27.79 & 7.09 & 3.97 & 0.14 & 2.36 & 2.11 & 0.96 & 1.76 & 0.26 & 0.52 & 0.25 & 76\end{array}$ $\begin{array}{llllllllllll}39.05 & 10.87 & 7.59 & 0.36 & 1.19 & 5.56 & 1.95 & 2.60 & 0.30 & 0.40 & 0.34 & 89\end{array}$ $\begin{array}{llllllllllll}43.23 & 8.68 & 9.62 & 0.46 & 1.62 & 6.19 & 1.89 & 1.66 & 0.28 & 0.36 & 0.24 & 35\end{array}$ $\begin{array}{lllllllllllll}30.98 & 10.56 & 4.72 & 0.18 & 0.54 & 4.17 & 3.06 & 0.90 & 0.70 & 0.19 & 0.17 & 95\end{array}$ $\begin{array}{llllllllllll}41.35 & 13.38 & 6.69 & 0.51 & 1.11 & 5.14 & 3.83 & 0.77 & 0.24 & 0.10 & 0.19 & 133\end{array}$ $\begin{array}{llllllllllll}35.49 & 7.66 & 7.97 & 0.32 & 0.93 & 5.75 & 1.74 & 0.31 & 0.46 & 0.07 & 0.22 & 110\end{array}$

10
0
0
0
0
0
0
0
0
0
0
51
0
0
7
0
0
0

$\begin{array}{lrrrrrr}0 & 89 & 44 & 36 & 39 & 17.58 & 86.92 \\ 0 & 79 & 29 & 38 & 40 & 17.00 & 88.65 \\ 0 & 74 & 11 & 25 & 30 & 23.92 & 85.64 \\ 0 & 64 & 35 & 26 & 24 & 16.33 & 87.13 \\ 0 & 85 & 35 & 20 & 22 & 7.75 & 86.17 \\ 0 & 104 & 15 & 14 & 19 & 8.83 & 90.01 \\ 0 & 89 & 35 & 24 & 36 & 26.17 & 88.38 \\ 0 & 77 & 6 & 17 & 17 & 20.00 & 85.83 \\ 0 & 71 & 48 & 26 & 34 & 45.17 & 95.59 \\ 0 & 78 & 20 & 20 & 27 & 30.00 & 91.66 \\ 0 & 2370 & 35 & 26 & 33 & 13.08 & 91.55 \\ 0 & 79 & 25 & 21 & 61 & 13.08 & 89.48 \\ 0 & 215 & 22 & 15 & 43 & 39.67 & 93.95 \\ 0 & 61 & 39 & 17 & 33 & 46.08 & 102.26 \\ 0 & 74 & 19 & 27 & 46 & 26.50 & 91.18 \\ 0 & 69 & 28 & 16 & 24 & 19.17 & 85.92 \\ 0 & 61 & 24 & 19 & 44 & 37.42 & 93.17 \\ 0 & 55 & 33 & 14 & 28 & 38.25 & 93.12\end{array}$

$\begin{array}{lllllll}0 & 55 & 33 & 14 & 28 & 38.25 & 93.12\end{array}$ 


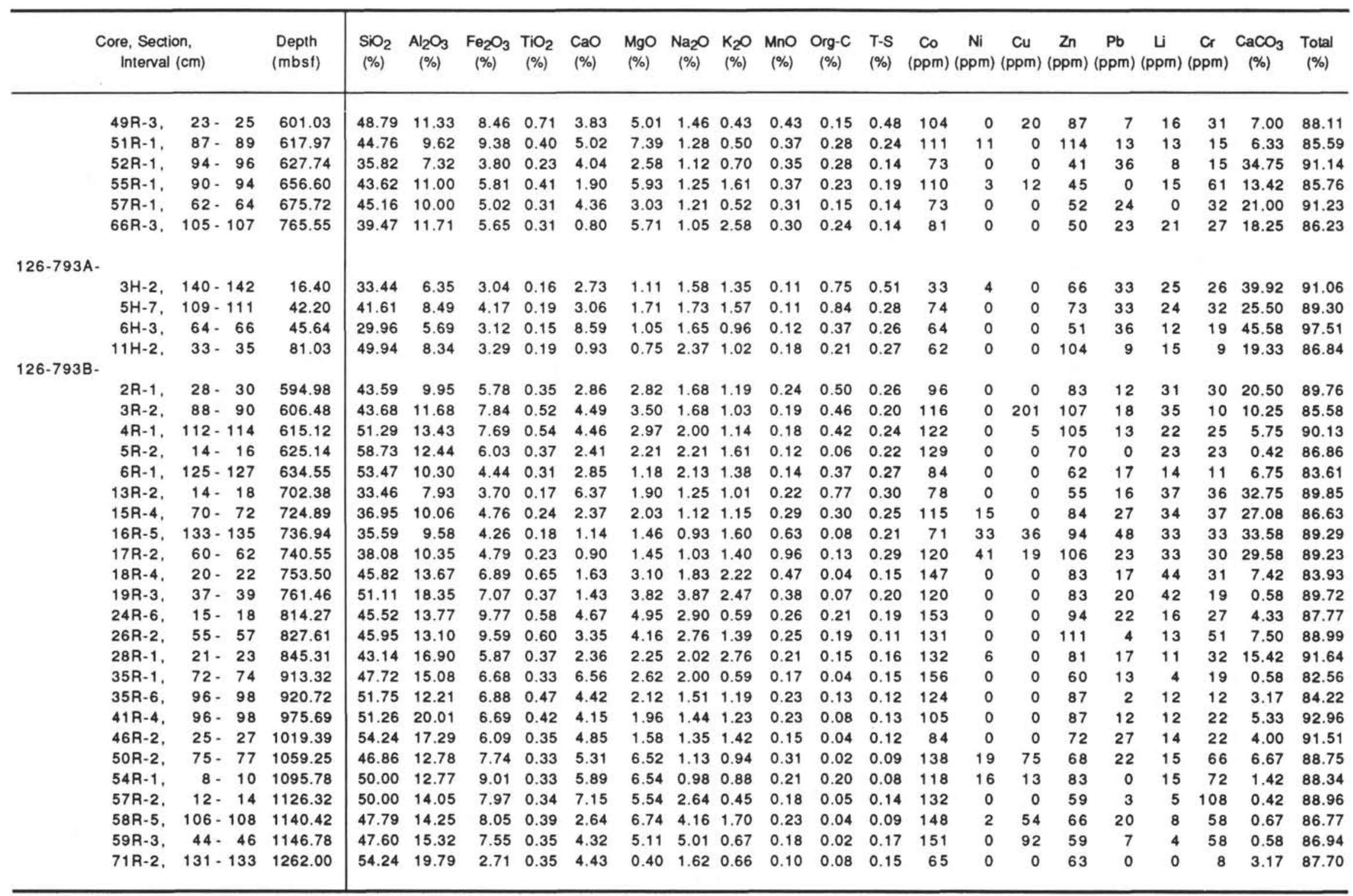

Note: Major elements in weight percent, and trace elements in parts per million (ppm). 


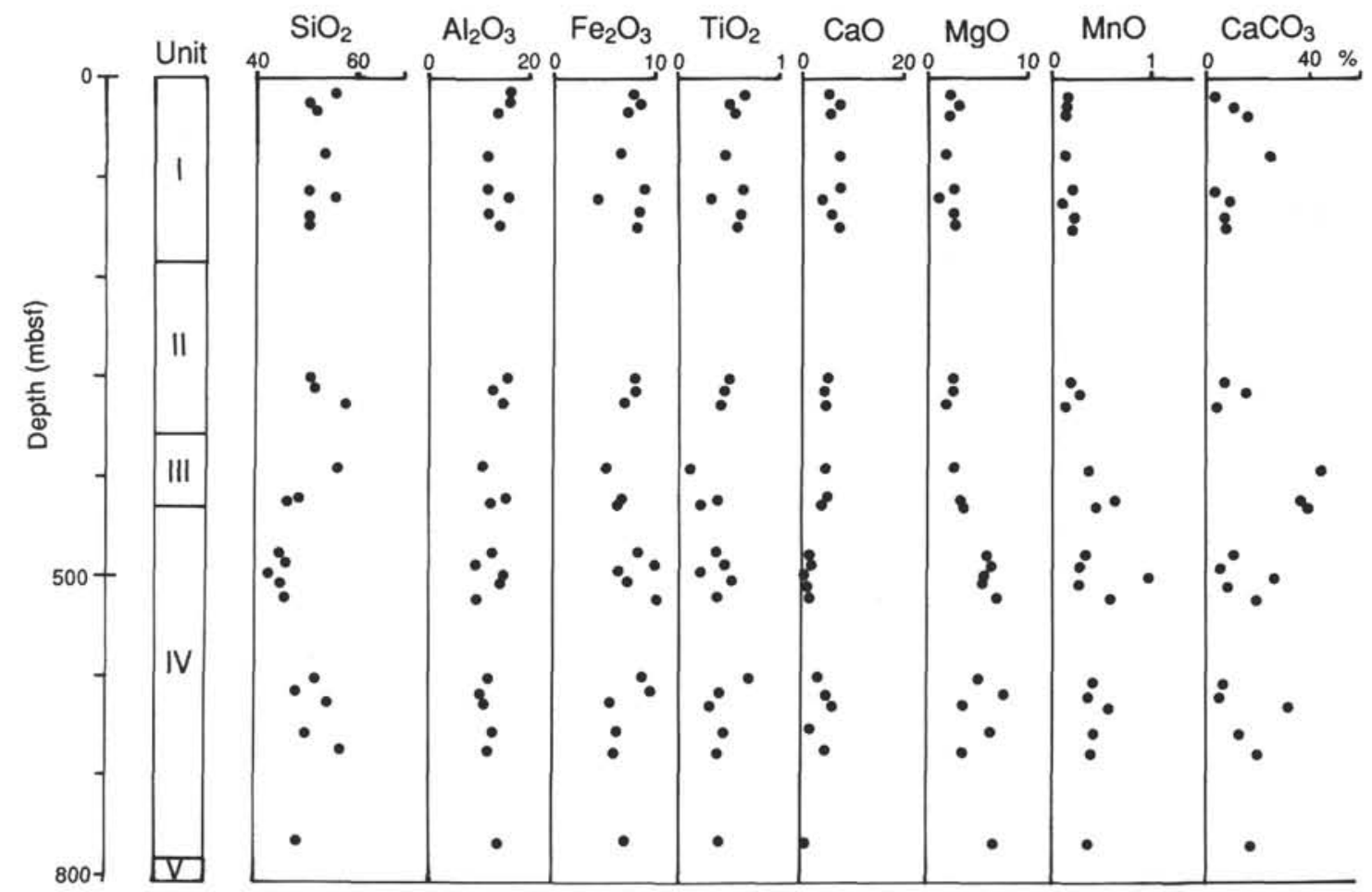

Figure 3. Vertical change of carbonate-free chemical compositions and carbonate content, Site 792.

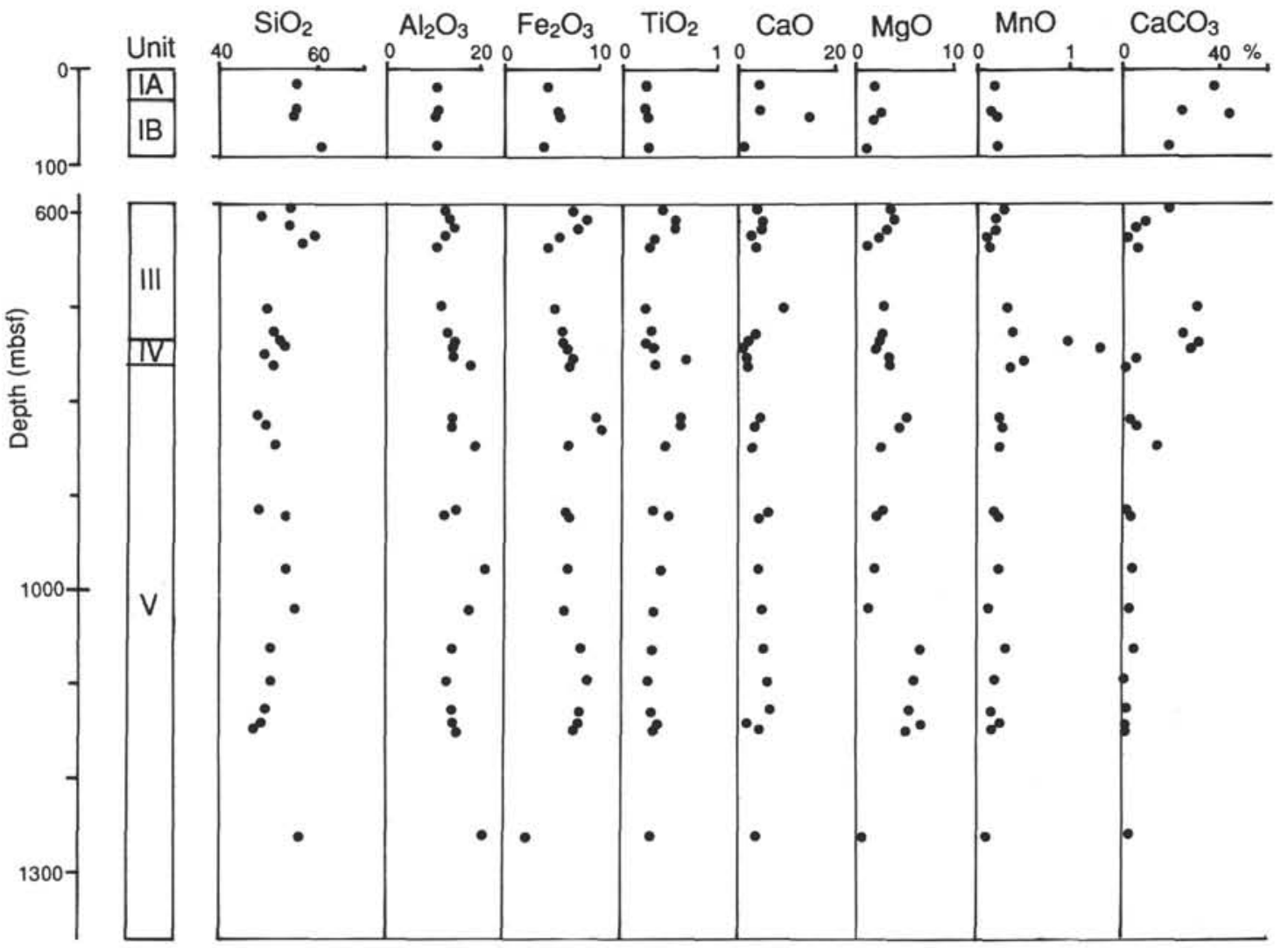

Figure 4. Vertical change of carbonate-free chemical compositions and carbonate content, Site 793. 
Table 2. Carbonate-free chemical compositions of pelagic and hemipelagic sediments of Leg 126, Izu-Bonin Arc.

\begin{tabular}{|c|c|c|c|c|c|c|c|c|c|c|c|c|c|}
\hline \multicolumn{3}{|c|}{$\begin{array}{l}\text { Core, Section, } \\
\text { Interval }(\mathrm{cm})\end{array}$} & \multirow{3}{*}{$\begin{array}{r}\begin{array}{r}\text { Depth } \\
\text { (mbsi) }\end{array} \\
\\
21.54\end{array}$} & \multirow{3}{*}{$\begin{array}{l}\mathrm{SiO}_{2} \\
(\%)\end{array}$} & \multirow{3}{*}{$\begin{array}{l}\begin{array}{c}\mathrm{Al}_{2} \mathrm{O}_{3} \\
(\%)\end{array} \\
12.56\end{array}$} & \multirow{3}{*}{$\begin{array}{r}\begin{array}{l}\mathrm{Fe}_{2} \mathrm{O}_{3} \\
(\%)\end{array} \\
8.05\end{array}$} & \multirow{3}{*}{$\begin{array}{l}\mathrm{TiO}_{2} \\
(\%) \\
0.69\end{array}$} & \multirow{3}{*}{$\begin{array}{l}\begin{array}{l}\mathrm{CaO} \\
(\%)\end{array} \\
4.82\end{array}$} & \multirow{3}{*}{$\begin{array}{l}\begin{array}{c}\mathrm{MgO} \\
(\%)\end{array} \\
2.58\end{array}$} & \multirow{3}{*}{$\begin{array}{l}\begin{array}{l}\mathrm{Na}_{2} \mathrm{O} \\
(\%)\end{array} \\
2.32\end{array}$} & \multirow{3}{*}{$\begin{array}{l}\mathrm{K}_{2} \mathrm{O} \\
(\%)\end{array}$} & \multirow{3}{*}{$\begin{array}{l}\begin{array}{c}\mathrm{MnO} \\
(\%)\end{array} \\
0.16\end{array}$} & \multirow{3}{*}{$\begin{array}{r}\begin{array}{r}\text { Total } \\
(\%)\end{array} \\
90.23\end{array}$} \\
\hline $126-787 \mathrm{~B}-$ & & & & & & & & & & & & & \\
\hline & $4 R-1$ & $14-19$ & & & & & & & & & & & \\
\hline & 6R-2, & $0-20$ & 41.55 & 60.05 & 10.76 & 5.85 & 0.36 & 2.00 & 1.81 & 2.35 & 2.00 & 0.25 & 86.14 \\
\hline & $8 R-1$ & $37-39$ & 59.67 & 54.24 & 11.19 & 6.61 & 0.41 & 14.04 & 2.67 & 2.09 & 2.12 & 0.62 & 97.66 \\
\hline & 9R-2, & $14 \cdot 15$ & 70.64 & 47.93 & 14.18 & 7.53 & 0.31 & 8.20 & 3.14 & 1.68 & 1.02 & 0.30 & 85.01 \\
\hline & 15R-4, & $64-67$ & 132.29 & 56.14 & 14.22 & 10.45 & 0.76 & 1.90 & 5.82 & 2.76 & 2.94 & 0.18 & 95.82 \\
\hline & 18R-2, & $32-36$ & 157.42 & 50.37 & 14.14 & 9.15 & 0.53 & 2.22 & 4.52 & 4.11 & 2.20 & 0.22 & 88.24 \\
\hline & 19R-6. & $89-91$ & 174.19 & 54.22 & 13.46 & 8.93 & 0.60 & 5.40 & 3.87 & 2.24 & 1.77 & 0.25 & 91.53 \\
\hline & 20R-2, & $70-74$ & 177.70 & 53.99 & 15.46 & 9.82 & 0.69 & 6.36 & 4.19 & 2.22 & 1.08 & 0.24 & 94.68 \\
\hline & 21R-4, & $52-55$ & 190.12 & 57.68 & 13.28 & 5.18 & 0.39 & 1.35 & 2.23 & 3.46 & 2.75 & 0.14 & 86.70 \\
\hline & 24R-7, & $36-40$ & 223.46 & 63.38 & 11.75 & 4.83 & 0.25 & 3.52 & 2.21 & 2.57 & 1.36 & .16 & 90.28 \\
\hline & 26R-6, & 7. 10 & 240.97 & 53.26 & 12.68 & 8.26 & 0.52 & 4.55 & 3.30 & 1.78 & 0.88 & 26 & 85.81 \\
\hline & $27 R-4$ & $26-28$ & 247.03 & 67.28 & 12.98 & 8.11 & 0.50 & 5.54 & 3.31 & 2.22 & 0.89 & 0.23 & 101.77 \\
\hline & 28R-2, & $25-28$ & 254.45 & 52.74 & 13.17 & 7.71 & 0.47 & 10.82 & 3.30 & 2.26 & 0.98 & 0.36 & 94.02 \\
\hline & $29 R-4$ & $121 \cdot 125$ & 268.11 & 53.68 & 14.31 & 7.99 & 0.40 & 11.69 & 3.04 & 2.08 & 1.18 & 0.27 & 96.80 \\
\hline & $30 R-2$ & $30-36$ & 273.80 & 51.87 & 11.95 & 6.55 & 0.38 & 15.32 & 2.85 & 2.11 & 1.25 & 0.22 & 94.42 \\
\hline & $32 \mathrm{R}-2$ & $90-95$ & 293.70 & 55.49 & 10.54 & 7.16 & 0.60 & 4.15 & 3.47 & 2.09 & 0.79 & 0.24 & 85.09 \\
\hline & $33 R-2$ & $107-110$ & 302.55 & 54.78 & 13.13 & 11.05 & 0.45 & 5.89 & 7.50 & 1.94 & 0.84 & 0.22 & 96.06 \\
\hline $126-788 \mathrm{D}-$ & & & & & & & & & & & & & \\
\hline & 5R-1. & $138-140$ & 259.58 & 53.26 & 11.01 & 11.25 & 0.89 & 4.09 & 4.65 & 1.95 & 1.15 & 0.29 & 89.2 \\
\hline & & & & & & & & & & & & & \\
\hline & $2 \mathrm{H}-1$, & $56-58$ & 9.36 & 64.48 & 11.37 & 5.98 & 0.56 & 4.15 & 1.77 & 3.26 & 1.34 & 0.17 & 94.88 \\
\hline & $2 \mathrm{H}-1$ & $112 \cdot 114$ & 9.92 & 64.18 & 12.17 & 6.40 & 0.47 & 2.32 & 2.44 & 2.85 & 2.34 & 0.17 & 95.98 \\
\hline $126-790 \mathrm{~B}-$ & & & & & & & & & & & & & \\
\hline & $7 \mathrm{H}-3$ & $50-60$ & 55.50 & 66.56 & 11.76 & 6.36 & 0.63 & 3.74 & 2.37 & 2.65 & 1.61 & 0.17 & 97.5 \\
\hline $126-790 \mathrm{C}-$ & $7-5$, & & & & & & & & & & & & \\
\hline & $10 X-2$ & $45-47$ & $\begin{array}{l}140.40 \\
166.46\end{array}$ & $\begin{array}{l}60.25 \\
60.74\end{array}$ & $\begin{array}{l}10.39 \\
11.90\end{array}$ & $\begin{array}{l}5.56 \\
5.59\end{array}$ & 0.50 & $\begin{array}{l}4.41 \\
4.60\end{array}$ & 2.50 & 2.18 & 2.36 & 0.17 & 92.92 \\
\hline & $12 X-1$ & $90-92$ & 185.10 & 57.70 & 12.40 & 7.99 & 0.59 & 7.83 & 2.94 & 2.25 & 1.21 & 0.23 & 95.10 \\
\hline & $16 \times-4$ & $129-149$ & 228.59 & 58.89 & 11.23 & 5.81 & 0.48 & 5.71 & 2.22 & 2.31 & 1.87 & 0.19 & 90.61 \\
\hline & $20 \times-4$ & $22 \cdot 24$ & 266.12 & 59.34 & 13.31 & 8.00 & 0.59 & 8.61 & 3.11 & 2.45 & 1.45 & 0.22 & 98.29 \\
\hline & & & & & & & & & & & & & \\
\hline & $4 \mathrm{H}-4$ & $130 \cdot 150$ & 29.30 & 56.36 & 10.57 & 5.35 & 0.48 & 5.03 & 2.23 & 2.33 & 1.96 & 0.14 & 87.12 \\
\hline & $16 \mathrm{H}-2$ & $92-94$ & 141.62 & 47.09 & & 4.46 & 0.38 & 11.61 & 1.86 & 2.04 & 1.70 & 12 & 79.44 \\
\hline & $18 \mathrm{H}-1$, & $70-72$ & 159.20 & 61.30 & 10.33 & 5.14 & 0.48 & 2.05 & 1.69 & 2.81 & 1.25 & 0.15 & 86.25 \\
\hline & $19 \mathrm{H}-1$ & $60-62$ & 168.70 & 62.09 & 10.18 & 4.93 & 0.52 & 2.44 & 1.57 & 3.19 & 1.05 & 0.16 & 86.64 \\
\hline & $22 \mathrm{H}-6$, & $45-65$ & 204.85 & 61.57 & 11.12 & 4.88 & 0.48 & 2.19 & 1.81 & 2.89 & 1.38 & 0.20 & 87.25 \\
\hline & $39 \times-1$ & $23-25$ & 352.53 & 57.89 & 10.00 & 4.66 & 0.54 & 3.61 & 1.45 & 2.63 & 2.14 & 0.16 & 84.46 \\
\hline 126-791B. & & & & & & & & & & & & & \\
\hline & 13R-1. & $97-99$ & 503.07 & 55.30 & 10.33 & 4.76 & 0.45 & 4.83 & 1.93 & 2.07 & 2.05 & 0.21 & 84.13 \\
\hline & $22 \mathrm{R}-1$ & $21 \cdot 22$ & 589.21 & 57.20 & 9.47 & 4.02 & 0.37 & 5.90 & 2.06 & 1.93 & 2.02 & 0.18 & 86.32 \\
\hline & $23 R-1$ & $125-126$ & 599.95 & 52.15 & 9.27 & 4.05 & 0.35 & 6.41 & 1.56 & 1.92 & 2.01 & 0.12 & 81.12 \\
\hline & 23R-4, & 6. 7 & 603.26 & 59.80 & 6.88 & 2.89 & 0.27 & 6.13 & 1.46 & 1.6 & 1.61 & & 84.62 \\
\hline & $24 R-1$ & $62-64$ & 609.02 & 56.14 & 11.07 & 6.05 & 0.49 & 4.05 & 2.46 & 2. & 36 & & 85.01 \\
\hline & 25R-2, & $51-52$ & 620.11 & 54.08 & & 8.2 & 0.64 & 6.40 & .17 & 8 & 33 & 21 & 89.05 \\
\hline & 25R-2, & $64 \cdot 66$ & 620.24 & 54.45 & 11.47 & 6.19 & 0.51 & 3.87 & .42 & 9 & 1.57 & 0.24 & 84.26 \\
\hline & $27 R-1$ & $30-31$ & 637.30 & 60.42 & 10.01 & 4.12 & 0.42 & 0.91 & 1.17 & 2.76 & 1.40 & 0.20 & 82.29 \\
\hline & $31 \mathrm{R}-3$ & $144-146$ & 680.04 & & 10.1 & 4.6 & 0.31 & 10.42 & 2.12 & 2.52 & 2.35 & 0.26 & 91.96 \\
\hline & $34 \mathrm{R}-2$ & $139-141$ & 707.49 & 63.58 & 9.6 & 3.61 & 0.27 & 3.05 & 1.47 & 2.61 & 2.11 & 0.19 & 88.09 \\
\hline & $38 \mathrm{R}-3$ & $110-112$ & 745.35 & 57.70 & 10.75 & 7.40 & 0.59 & 5.42 & 2.74 & 2.26 & 1.58 & 0.22 & 90.28 \\
\hline & $39 R-2$, & $45-46$ & 754.35 & 49.73 & 10.71 & 6.95 & 0.58 & 9.70 & 3.30 & 1.98 & 1.36 & 0.16 & 87.90 \\
\hline & 40R-3, & $10-11$ & 765.20 & 51.99 & 10.13 & 5.59 & 0.48 & 12.99 & 2.54 & 2.47 & 1.71 & 0.30 & 89.98 \\
\hline & 41R-3, & $77-79$ & 775.47 & 51.99 & 10.26 & 6.27 & 0.37 & 26.15 & 2.58 & 2.32 & 1.89 & 0.24 & 104.20 \\
\hline & $42 \mathrm{R}-2$ & $72-73$ & 783.52 & 55.58 & 11.09 & 5.81 & 0.52 & 6.45 & 2.65 & 2.19 & 1.99 & 0.16 & 88.00 \\
\hline & 43R-1, & $31-32$ & 791.31 & 59.44 & 7.48 & 4.65 & 0.36 & 0.97 & 0.00 & 1.58 & 2.61 & 0.12 & 82.58 \\
\hline & $43 R-1$ & $136-137$ & 792.36 & 58.27 & 11.12 & 6.20 & 0.46 & 4.60 & 2.92 & 2.33 & 1.87 & 0.42 & 89.09 \\
\hline & 46R-2 & $34-36$ & 821.84 & 59.16 & 10.62 & 5.34 & 0.32 & 5.36 & 2.11 & 2.57 & 1.72 & 0.23 & 88.86 \\
\hline & & & & & & & & & & & & & \\
\hline & $2 \mathrm{H}-3$, & $140-142$ & 14.10 & 56.35 & 16.24 & 8.11 & 0.66 & 5.32 & 2.52 & 2.50 & 0.84 & 0.19 & 93.25 \\
\hline & $3 \mathrm{H}-6$, & $72-92$ & 27.32 & 51.38 & 16,42 & 8.48 & 0.56 & 7.42 & 3.23 & 2.12 & 0.95 & 0.17 & 91.79 \\
\hline
\end{tabular}


Table 2 (continued).

\begin{tabular}{|c|c|c|c|c|c|c|c|c|c|c|c|c|c|}
\hline & $4 \mathrm{H}-6$, & $100-102$ & 36.90 & 51.71 & 13.78 & 7.32 & 0.54 & 5.51 & 2.29 & 2.28 & 1.09 & 0.15 & 85.80 \\
\hline & $9 \mathrm{H}-2$, & $19-21$ & 77.01 & 54.06 & 11.37 & 6.77 & 0.49 & 7.34 & 2.13 & 2.68 & 1.26 & 0.16 & 87.49 \\
\hline & $8 \times-1$, & $112 \cdot 114$ & 109.02 & 51.89 & 14.69 & 9.15 & 0.68 & 6.69 & 2.65 & 2.25 & 0.69 & 0.20 & 89.65 \\
\hline & $9 \times-2$, & $13-15$ & 119.13 & 56.51 & 11.82 & 4.60 & 0.32 & 4.12 & 1.36 & 2.66 & 1.30 & 0.14 & 83.82 \\
\hline \multicolumn{14}{|l|}{ 126-792E- } \\
\hline & $\begin{array}{l}\text { 1R-1, } \\
2 R-1,\end{array}$ & $\begin{array}{rr}17-\quad 19 \\
7-\quad 9\end{array}$ & $\begin{array}{l}135.77 \\
145.27\end{array}$ & $\begin{array}{l}50.51 \\
51.64\end{array}$ & $\begin{array}{l}13.27 \\
14.48\end{array}$ & $\begin{array}{l}8.93 \\
8.50\end{array}$ & $\begin{array}{l}0.64 \\
0.59\end{array}$ & 7.00 & $\begin{array}{l}2.87 \\
2.68\end{array}$ & $\begin{array}{l}2.21 \\
2.25\end{array}$ & $\begin{array}{l}0.78 \\
0.81\end{array}$ & $\begin{array}{l}0.19 \\
0.20\end{array}$ & $\begin{array}{l}86.57 \\
88.97\end{array}$ \\
\hline & $18 \mathrm{R}-1$, & $70-72$ & 300.20 & 51.10 & 13.07 & 8.30 & 0.52 & 5.18 & 2.76 & 2.35 & 1.03 & 0.20 & $\begin{array}{l}88.91 \\
84.95\end{array}$ \\
\hline & $19 R-2$. & $120-122$ & 311.70 & 51.45 & 12.79 & 8.31 & 0.48 & 4.77 & 2.70 & 2.18 & 0.89 & 0.30 & 84.21 \\
\hline & 21R-1, & $34-35$ & 328.64 & 58.02 & 15.34 & 7.12 & 0.43 & 5.09 & 2.12 & 2.22 & 0.89 & 0.22 & 91.77 \\
\hline & 27R-3, & $100-102$ & 389.42 & 56.75 & 11.64 & 5.42 & 0.11 & 5.13 & 2.75 & 2.36 & 1.98 & 0.41 & 88.24 \\
\hline & $30 R-4$, & $30-50$ & 420.00 & 49.10 & 12.06 & 7.05 & 0.40 & 5.31 & 3.50 & 1.79 & 4.08 & 0.66 & 85.43 \\
\hline & 31R-1, & $57-59$ & 425.47 & 47.64 & 12.15 & 6.81 & 0.24 & 4.04 & 3.62 & 1.65 & 3.02 & 0.45 & 80.96 \\
\hline & $36 R-4$. & $2-5$ & 477.72 & 44.76 & 12.46 & 8.70 & 0.41 & 1.36 & 6.37 & 2.23 & 2.98 & 0.34 & 80.50 \\
\hline & 37R-2, & $62-63$ & 484.92 & 46.32 & 9.30 & 10.31 & 0.49 & 1.74 & 6.63 & 2.02 & 1.78 & 0.30 & 79.56 \\
\hline & $38 \mathrm{R}-2$, & $84-87$ & 494.84 & 43.63 & 14.87 & 6.65 & 0.25 & 0.76 & 5.87 & 4.31 & 1.27 & 0.99 & 79.12 \\
\hline & 39R-3, & $1-3$ & 505.21 & 44.90 & 14.53 & 7.27 & 0.55 & 1.21 & 5.58 & 4.16 & 0.84 & 0.26 & 79.64 \\
\hline & 4OR-5, & $85-87$ & 518.75 & 45.89 & 9.91 & 10.31 & 0.41 & 1.20 & 7.44 & 2.25 & 0.40 & 0.59 & 78.82 \\
\hline & 49R-3, & $23-25$ & 601.03 & 52.46 & 12.18 & 9.10 & 0.76 & 4.12 & 5.39 & 1.57 & 0.46 & 0.46 & 87.21 \\
\hline & 51R-1, & $87-89$ & 617.97 & 47.79 & 10.27 & 10.01 & 0.43 & 5.36 & 7.89 & 1.37 & 0.53 & 0.40 & 84.62 \\
\hline & 52R-1. & $94-96$ & 627.74 & 54.90 & 11.22 & 5.82 & 0.35 & 6.19 & 3.95 & 1.72 & 1.07 & 0.54 & 86.42 \\
\hline & 55R-1, & $90-94$ & 656.60 & 50.38 & 12.70 & 6.71 & 0.47 & 2.20 & 6.85 & 1.44 & 1.86 & 0.43 & 83.55 \\
\hline & 57R-1. & $62-64$ & 675.72 & 57.16 & 12.66 & 6.35 & 0.39 & 5.52 & 3.84 & 1.53 & 0.66 & 0.39 & 88.90 \\
\hline & 66R-3, & $105-107$ & 765.55 & 48.28 & 14.32 & 6.91 & 0.38 & 0.98 & 6.98 & 1.28 & 3.16 & 0.37 & 83.16 \\
\hline \multicolumn{14}{|l|}{$126.793 \mathrm{~A}-$} \\
\hline & $3 \mathrm{H}-2$, & $140-142$ & 16.40 & 55.65 & 10.57 & 5.06 & 0.27 & 4.54 & 1.85 & 2.63 & 2.25 & 0.18 & 85.12 \\
\hline & $5 \mathrm{H}-7$. & $109-111$ & 42.20 & 55.85 & 11.40 & 5.60 & 0.26 & 4.11 & 2.30 & 2.32 & 2.11 & 0.15 & 85.64 \\
\hline & $6 \mathrm{H}-3$, & $64-66$ & 45.64 & 55.05 & 10.46 & 5.73 & 0.28 & 15.79 & 1.93 & 3.03 & 1.76 & 0.22 & 95.43 \\
\hline & $11 \mathrm{H}-2$, & $33-35$ & 81.03 & 61.91 & 10.34 & 4.08 & 0.24 & 1.15 & 0.93 & 2.94 & 1.26 & 0.22 & 83.69 \\
\hline \multicolumn{14}{|l|}{$126.793 B$ - } \\
\hline & $\begin{array}{l}2 R-1, \\
3 R-2,\end{array}$ & $\begin{array}{l}28-30 \\
88-90\end{array}$ & $\begin{array}{l}594.98 \\
606.48\end{array}$ & 48.67 & 13.01 & 8.74 & 0.58 & 5.00 & 3.90 & 1.87 & 1.15 & 0.21 & $\begin{array}{l}87.12 \\
83.93\end{array}$ \\
\hline & $4 R-1$, & $112-114$ & 615.12 & 54.42 & 14.25 & 8.16 & 0.57 & 4.73 & 3.15 & 2.12 & 1.21 & 0.19 & 89.53 \\
\hline & $5 R-2$, & $14-16$ & 625.14 & 58.98 & 12.49 & 6.06 & 0.37 & 2.42 & 2.22 & 2.22 & 1.62 & 0.12 & 86.81 \\
\hline & $6 R-1$ & $125-127$ & 634.55 & 57.34 & 11.05 & 4.76 & 0.33 & 3.05 & 1.27 & 2.28 & 1.48 & 0.15 & 82.42 \\
\hline & 13R-2, & $14-18$ & 702.38 & 49.75 & 11.79 & 5.50 & 0.25 & 9.47 & 2.83 & 1.86 & 1.50 & 0.33 & 84.91 \\
\hline & $15 \mathrm{R}-4$, & $70-72$ & 724.89 & 50.67 & 13.80 & 6.53 & 0.33 & 3.24 & 2.78 & 1.54 & 1.58 & 0.40 & 81.66 \\
\hline & 16R-5. & $133-135$ & 736.94 & 53.58 & 14.42 & 6.41 & 0.27 & 1.72 & 2.20 & 1.40 & 2.41 & 0.95 & 83.87 \\
\hline & 17R-2, & $60-62$ & 740.55 & 54.08 & 14.70 & 6.80 & 0.33 & 1.27 & 2.06 & 1.46 & 1.99 & 1.36 & 84.70 \\
\hline & 18R-4, & $20-22$ & 753.50 & 49.49 & 14.77 & 7.44 & 0.70 & 1.77 & 3.35 & 1.98 & 2.40 & 0.51 & 82.64 \\
\hline & 19R-3, & $37-39$ & 761.46 & 51.41 & 18.46 & 7.11 & 0.37 & 1.44 & 3.84 & 3.89 & 2.48 & 0.38 & 89.66 \\
\hline & 24R-6, & 15. 18 & 814.27 & 47.58 & 14.39 & 10.21 & 0.61 & 4.88 & 5.17 & 3.03 & 0.62 & 0.27 & 87.21 \\
\hline & 26R-2. & $55-57$ & 827.61 & 49.68 & 14.16 & 10.37 & 0.65 & 3.62 & 4.50 & 2.98 & 1.50 & 0.27 & 88.10 \\
\hline & 28R-1, & 21. 23 & 845.31 & 51.00 & 19.98 & 6.94 & 0.44 & 2.79 & 2.66 & 2.39 & 3.26 & 0.25 & 90.12 \\
\hline & $35 R-1$. & $72-74$ & 913.32 & 48.00 & 15.17 & 6.72 & 0.33 & 6.60 & 2.64 & 2.01 & 0.59 & 0.17 & 82.45 \\
\hline & $35 R-6$, & $96 \cdot 98$ & 920.72 & 53.44 & 12.61 & 7.10 & 0.49 & 4.56 & 2.19 & 1.56 & 1.23 & 0.24 & 83.71 \\
\hline & $41 R-4$. & $96-98$ & 975.69 & 54.15 & 21.14 & 7.07 & 0.44 & 4.39 & 2.07 & 1.52 & 1.30 & 0.24 & 92.56 \\
\hline & 46R-2, & $25-27$ & 1019.39 & 56.50 & 18.01 & 6.34 & 0.36 & 5.05 & 1.65 & 1.41 & 1.48 & 0.16 & 91.16 \\
\hline & 50R-2, & $75-77$ & 1059.25 & 50.21 & 13.69 & 8.29 & 0.35 & 5.69 & 6.99 & 1.21 & 1.01 & 0.33 & 87.95 \\
\hline & 54R-1, & $8-10$ & 1095.78 & 50.72 & 12.95 & 9.14 & 0.33 & 5.97 & 6.63 & 0.99 & 0.89 & 0.21 & 88.18 \\
\hline & 57R-2, & $12-14$ & 1126.32 & 50.21 & 14.11 & 8.00 & 0.34 & 7.18 & 5.56 & 2.65 & 0.45 & 0.18 & 88.92 \\
\hline & 58R-5. & $106-108$ & 1140.42 & 48.11 & 14.35 & 8.10 & 0.39 & 2.65 & 6.79 & 4.19 & 1.71 & 0.23 & 86.68 \\
\hline & 59R-3, & $44-46$ & 1146.78 & 47.88 & 15.41 & 7.59 & 0.35 & 4.35 & 5.14 & 5.04 & 0.67 & 0.18 & 86.86 \\
\hline & 71R-2, & $131-133$ & 1262.00 & 56.01 & 20.44 & 2.80 & 0.36 & 4.57 & 0.41 & 1.67 & 0.68 & 0.10 & 87.30 \\
\hline
\end{tabular}

Note: Major elements in weight percent.

calcareous nannofossils throughout the sequences. Biogenic siliceous components, such as diatoms, radiolarians, and sponge spicules, are few except in several horizons that contain up to $10 \%$ of them. Therefore, the biogenic components of hemipelagic and pelagic sediments are thought to contribute mainly to the calcium carbonate content of sediments in the Izu-Bonin area. Unit V of Site 793 is thought to have been deposited below the calcium carbonate compensation depth (CCD), and other sedimentary sequences show deposition above the CCD based on the benthic foraminifer assemblages
(Kaiho, this volume). The calcium carbonate contents of Unit V of Site 793 are $<7 \%$, except for one sample that reaches $15 \%$. On the contrary, the calcium carbonate contents of the sediments of other units are variable and attain values as high and higher than $45 \%$ (Table 1).

The Sr isotopic ratios of carbonates from the sediments of Unit III of Site 792 are lower in value than those of modern marine carbonates, as already mentioned. These values yield ages of $23.5 \mathrm{Ma}$ for Sample 126-792E-28R-1, 57-59 cm, and $28.4 \mathrm{Ma}$ for Sample 126-792E$31 \mathrm{R}-1,31-32 \mathrm{~cm}$, based on the data of Hess et al. (1986). The 
Table 3. Averages and standard deviations of carbonate-free chemical compositions of each unit of the sites drilled on Leg 126, Izu-Bonin Arc.

\begin{tabular}{|c|c|c|c|c|c|c|c|c|}
\hline $\begin{array}{l}\text { Site } \\
\text { Unit }\end{array}$ & $\begin{array}{c}\text { Site } 787 \\
\text { Unit II }\end{array}$ & $\begin{array}{l}\text { Site } 787 \\
\text { Unit III }\end{array}$ & $\begin{array}{ll}\text { Site } & 787 \\
\text { Unit IVA }\end{array}$ & $\begin{array}{ll}\text { Site } & 787 \\
\text { Unit IVB }\end{array}$ & $\begin{array}{l}\text { Site } 788 \\
\text { Unit } \| A\end{array}$ & $\begin{array}{c}\text { Site } 790 \\
\text { Unit I }\end{array}$ & $\begin{array}{c}\text { Site } 790 \\
\text { Unit II }\end{array}$ & $\begin{array}{c}\text { Site } 791 \\
\text { Unit I }\end{array}$ \\
\hline Samples (n) & 1 & 3 & 11 & 2 & 1 & 4 & 4 & 6 \\
\hline $\mathrm{SiO}_{2}$ & 56.62 & 54.07 & $55.87 \pm 5.14$ & 55.14 & 53.26 & $63.87 \pm 2.64$ & $59.17 \pm 1.26$ & $57.72 \pm 5.68$ \\
\hline $\mathrm{Al}_{2} \mathrm{O}_{3}$ & 12.56 & 12.04 & $13.40 \pm 1.09$ & 11.83 & 11.01 & $11.42 \pm 0.76$ & $12.21 \pm 0.88$ & $10.18 \pm 0.74$ \\
\hline $\mathrm{Fe}_{2} \mathrm{O}_{3}$ & 8.05 & 6.66 & $7.91 \pm 1.78$ & 9.1 & 11.25 & $6.08 \pm 0.39$ & $6.85 \pm 1.33$ & $4.90 \pm 0.32$ \\
\hline $\mathrm{TiO}_{2}$ & 0.69 & 0.36 & $0.50 \pm 0.15$ & 0.53 & 0.89 & $0.52 \pm 0.10$ & $0.54 \pm 0.06$ & $0.48 \pm 0.05$ \\
\hline $\mathrm{CaO}$ & 4.28 & 8.08 & $6.24 \pm 4.51$ & 5.02 & 4.09 & $3.65 \pm 0.93$ & $6.68 \pm 1.85$ & $4.49 \pm 3.67$ \\
\hline $\mathrm{MgO}$ & 2.58 & 2.54 & $3.51 \pm 1.05$ & 5.48 & 4.65 & $2.10 \pm 0.35$ & $2.69 \pm 0.41$ & $1.77 \pm 0.27$ \\
\hline $\mathrm{Na}_{2} \mathrm{O}$ & 2.32 & 2.04 & $2.53 \pm 0.68$ & 2.02 & 1.95 & $2.82 \pm 0.32$ & $2.30 \pm 0.11$ & $2.65 \pm 0.41$ \\
\hline $\mathrm{K}_{2} \mathrm{O}$ & 1.19 & 1.71 & $1.57 \pm 1.45$ & 0.82 & 1.15 & $1.68 \pm 0.45$ & $1.72 \pm 0.51$ & $1.58 \pm 0.42$ \\
\hline $\mathrm{MnO}$ & 0.16 & 0.39 & $0.23 \pm 0.06$ & 0.23 & 0.29 & $0.18 \pm 0.02$ & $0.20 \pm 0.03$ & $0.15 \pm 0.03$ \\
\hline
\end{tabular}

Notes: Major elements in weight percent. $(\mathrm{n})=$ number of samples analyzed.

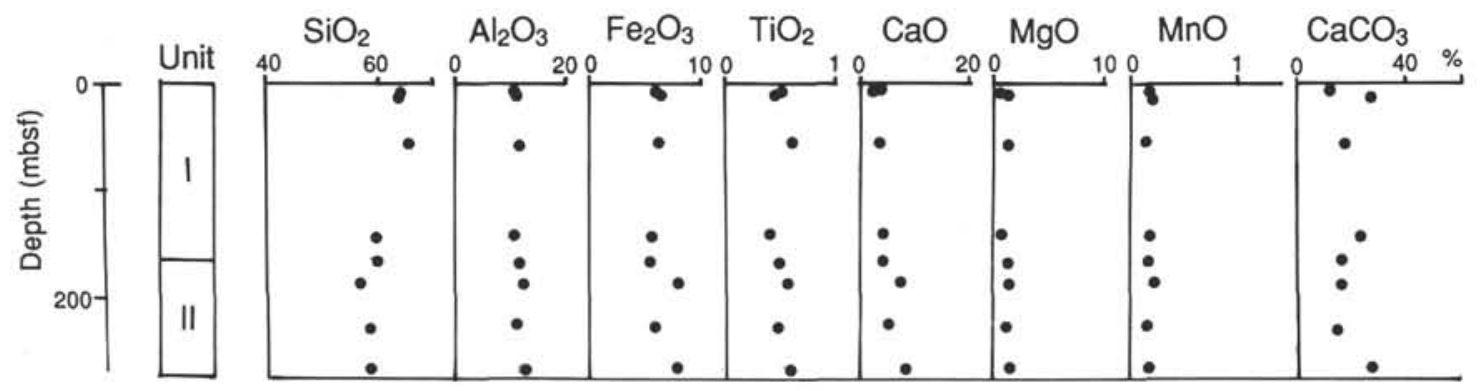

Figure 5. Vertical change of carbonate-free chemical compositions and carbonate content, Site 790.

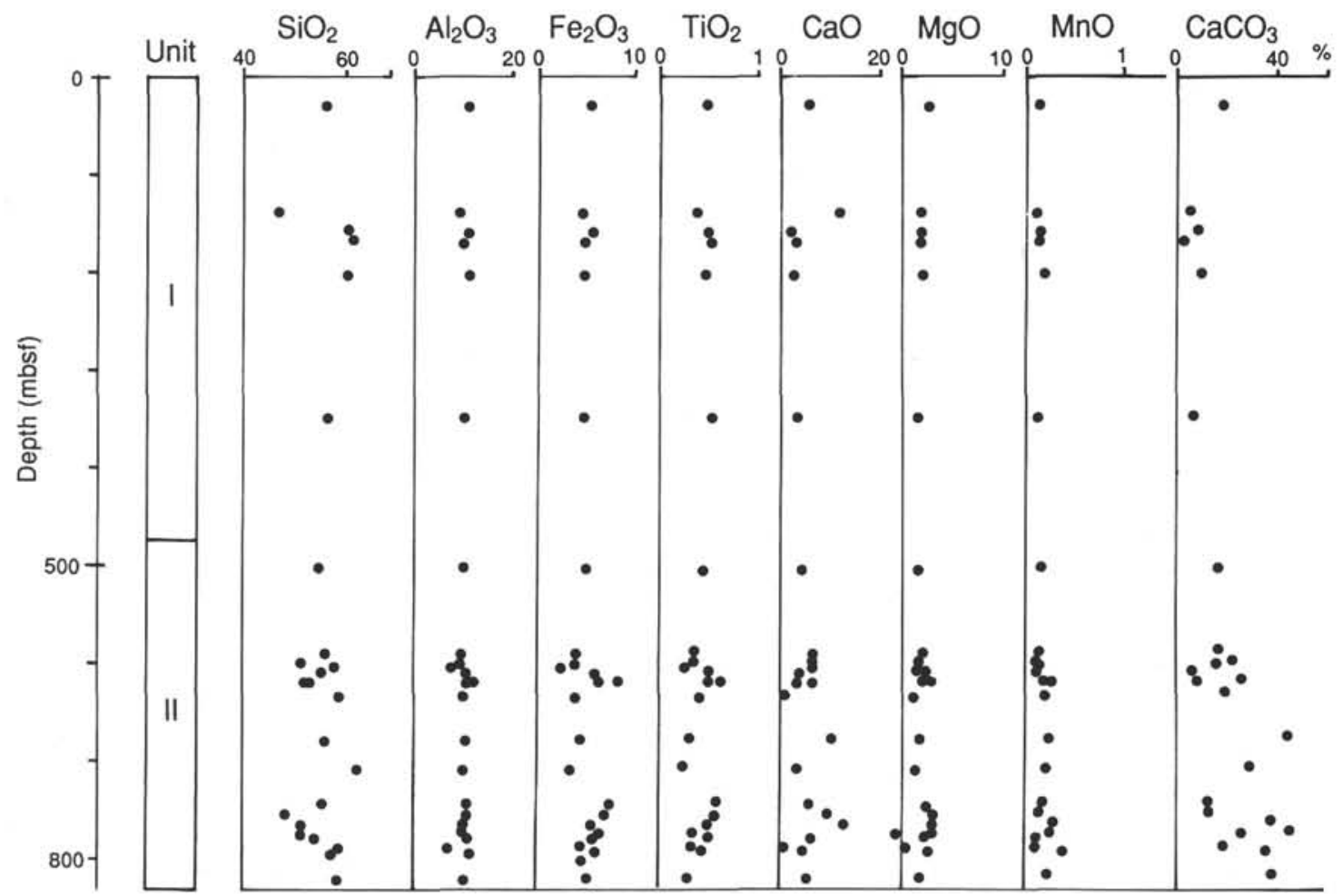

Figure 6. Vertical change of carbonate-free chemical compositions and carbonate content, Site 791. 
Table 3 (continued).

\begin{tabular}{|c|c|c|c|c|c|c|c|c|c|}
\hline $\begin{array}{c}\text { Site } 791 \\
\text { Unit II }\end{array}$ & $\begin{array}{c}\text { Site } 792 \\
\text { Unit I }\end{array}$ & $\begin{array}{c}\text { Site } 792 \\
\text { Unit II }\end{array}$ & $\begin{array}{l}\text { Site } 792 \\
\text { Unit III }\end{array}$ & $\begin{array}{l}\text { Site } 792 \\
\text { Unit IV }\end{array}$ & $\begin{array}{c}\text { Site } 793 \\
\text { Unit IA }\end{array}$ & $\begin{array}{c}\text { Site } 793 \\
\text { Unit IB }\end{array}$ & $\begin{array}{l}\text { Site } 793 \\
\text { Unit III }\end{array}$ & $\begin{array}{l}\text { Site } 793 \\
\text { Unit IV }\end{array}$ & $\begin{array}{c}\text { Site } 793 \\
\text { Unit V }\end{array}$ \\
\hline 18 & 8 & 3 & 3 & 11 & 1 & 3 & 7 & 3 & 14 \\
\hline $56.33 \pm 3.56$ & $53.01 \pm 2.34$ & 53.52 & 51.16 & $48.77 \pm 4.44$ & 55.65 & 57.61 & $53.52 \pm 3.93$ & 52.38 & $51.06 \pm 2.95$ \\
\hline $10.14 \pm 1.29$ & $14.01 \pm 1.84$ & 13.74 & 11.95 & $12.22 \pm 1.90$ & 10.57 & 10.73 & $12.70 \pm 1.11$ & 14.63 & $16.06 \pm 2.93$ \\
\hline $5.38 \pm 1.40$ & $7.73 \pm 1.50$ & 7.91 & 6.42 & $8.01 \pm 1.70$ & 5.06 & 5.14 & $6.72 \pm 1.43$ & 6.89 & $7.56 \pm 1.85$ \\
\hline $0.43 \pm 0.11$ & $0.56 \pm 0.12$ & 0.48 & 0.25 & $0.45 \pm 0.13$ & 0.27 & 0.26 & $0.41 \pm 0.13$ & 0.43 & $0.42 \pm 0.10$ \\
\hline $6.87 \pm 5.68$ & $6.22 \pm 1.16$ & 5.01 & 4.83 & $2.79 \pm 2.08$ & 4.54 & 7.02 & $4.50 \pm 2.38$ & 1.59 & $4.55 \pm 1.57$ \\
\hline $2.15 \pm 0.80$ & $2.47 \pm 0.56$ & 2.53 & 3.29 & $6.07 \pm 1.31$ & 1.85 & 1.72 & $2.81 \pm 0.87$ & 2.54 & $4.02 \pm 2.11$ \\
\hline $2.22 \pm 0.32$ & $2.37 \pm 0.21$ & 2.25 & 1.93 & $2.17 \pm 1.07$ & 2.63 & 2.76 & $2.00 \pm 0.26$ & 1.61 & $2.47 \pm 1.23$ \\
\hline $1.78 \pm 0.41$ & $0.97 \pm 0.23$ & 0.93 & 3.03 & $1.36 \pm 0.98$ & 2.25 & 1.71 & $1.43 \pm 0.18$ & 2.26 & $1.28 \pm 0.79$ \\
\hline $0.21 \pm 0.07$ & $0.17 \pm 0.02$ & 0.24 & 0.51 & $0.46 \pm 0.20$ & 0.18 & 0.2 & $0.24 \pm 0.10$ & 0.94 & $0.23 \pm 0.07$ \\
\hline
\end{tabular}

magnetostratigraphy and biostratigraphy yield ages of $22.6-23.5 \mathrm{Ma}$ and 27.0-27.5 Ma for the two samples, respectively. This coincidence indicates pelagic sedimentation of calcium carbonate in this unit.

\section{Terrigenous Components in Chemical Composition}

In the $\mathrm{Fe}_{2} \mathrm{O}_{3}-\mathrm{MgO}-\mathrm{TiO}_{2}$ triangles, the Oligocene sequences of forearc sites show high contents of $\mathrm{MgO}$, compared with those of the other sedimentary sequences (Fig. 8). At Site 792, the chemical compositions are clearly separated in the triangle and are arranged from the Quaternary unit (with low $\mathrm{MgO}$ contents) to the Oligocene unit (with high $\mathrm{MgO}$ contents). The $\mathrm{MgO}$ content of the Oligocene sequence is higher, with some samples exceeding 7\% (Table 2). High magnesian boninitic and andesitic rocks with $\mathrm{MgO}$ contents exceeding $20 \%$ occur in the Chichijima Island of the Ogasawara (Bonin) Ridge (Kuroda and Shiraki, 1975; Umino, 1985). The Oligocene basement volcanic rock sequences of forearc Sites 792 and 793 attain $\mathrm{MgO}$ contents up to $12 \%$ (Taylor, Fujioka, et al., 1990). In contrast, $\mathrm{MgO}$ contents in the late Miocene to Quaternary sequences of the forearc sites are as small as those of the Quaternary sequences at the backarc sites, which are lower than $4 \%$ (Table 2).

The sedimentation rates (even the net sedimentation rates of muddy sediments) of the Oligocene sequences were calculated to several tens to hundred meters per million years, except for the late Oligocene to early Miocene Unit III of Site 792 and Unit IV of Site 793, both of which have very slow rates (e.g., $4-14 \mathrm{~m} / \mathrm{m}$.y.). The sedimentation rates of the Oligocene sequences are distinctly larger than those in oceanic pelagic environments (Table 5), which suggests that the contribution of terrigenous inputs to hemipelagic sediments is probably several times as large as those in pelagic environments. Therefore, the chemical composition of hemipelagic sediments directly reflects those of the source area. The $\mathrm{MgO}$ enrichment in the Oligocene sequences of the forearc sites shows the influence of the chemical composition of the immature Paleogene arc with high magnesian rock series to those of the hemipelagic sediments.

A similar relationship was found in the coarser grained sediments. The chemical composition of sandstone in the Oligocene sequences of the forearc shows the same tendency, with the high $\mathrm{MgO}$ contents reflected in those of the source area (Hiscott and Gill, this volume).

The REE patterns of the sediments in Figures 9 and 10 show that the absolute abundance of REEs in the sediments is generally higher than that of oceanic basalts and that all of the sediments are enriched in light REEs. Enrichment in light REEs in the sediments is more marked in the backarc than in the forearc. Ikeda and Yuasa (1989) showed that volcanic rocks from the Sumisu Rift and its vicinity generally have two types of REE patterns, that is, enriched $(E)$ and depleted $(\mathrm{N})$ types. Most of the volcanic rocks from the arc ridge and the intraridge in the Sumisu Rift possess an N-type pattern and are depleted to moderate in light REEs with $0.66-1.50(\mathrm{Ce} / \mathrm{Yb})_{\mathrm{n}}$ (Ikeda and Yuasa, 1989; Freyer et al., 1990; Hochstaedter et al., 1990). However, volcanic rocks from the backarc seamounts northwest of the Sumisu Rift display an E-type pattern with 1.7-3.23 (Ce/Yb) (Ikeda and Yuasa, 1989). The sediment samples from the Sumisu Rift exhibit strongly light-REE-enriched patterns with $2.31-4.33(\mathrm{Ce} / \mathrm{Yb})_{n}$, suggesting that they probably originated from the enriched-type rocks (Figs. 9 and 11). The $\mathrm{MgO}$ contents of the sediments suggests that forearc sediments are highly affected by high magnesian rocks, as mentioned above. However, the REE data of the forearc sediments displays a high abundance and a different pattern from the boninitic rocks. Boninites from the Bonin Island contain a very low abundance in REEs with $0.88(\mathrm{Ce} / \mathrm{Yb})_{\mathrm{n}}$ and a dish-shaped pattern (Hickey and Frey, 1982) (Fig. 11). To know the origin of the difference in the REE pattern between the backarc and forearc sediments, more work focused on the sedimentary environment and the sedimentary process of hemipelagic sediments is needed.

\section{Hydrothermal Effects on Chemical Composition in the Sumisu Rift}

In the Izu-Bonin Arc, crusts of manganese oxides with hydrothermal origins were found on the slope area of active volcanoes along the volcanic front (Usui et al., 1986; Nakao et al., 1990). In the Sumisu Rift, high manganese contents of surface hemipelagic sediments exceeding $1 \%$ are characteristic (Nishimura et al., 1988; Nakao et al., 1990). Moreover, on the ridges between the north and south basins of the Sumisu Rift, dead but very young chimneys composed of silica and barites were found (Taylor et al., 1990), which can be a source of manganese in the surface sediments of the Sumisu Rift basin (Urabe and Kusakabe, 1990). In this study, however, the MnO contents in the sediments of the Sumisu Rift are low, with the highest value at only $0.4 \%$ (Table 1). This suggests that the hydrothermal influence on the chemical composition of hemipelagic sediments is negligible throughout the core sequences of Sites 790 and 791. It should be pointed out that manganese ions were released from the bottom through the diagenetic reductive processes, which are related to the higher organic carbon contents of sediments and the higher sedimentation rate in the Sumisu Rift. Surface sediments of the Sumisu Rift with high $\mathrm{MnO}$ contents have a dark brown color (Nishimura and Murakami, 1988), but the samples analyzed in this study possess an olive gray color. The REE analyses of the samples at Site 791 also do not indicate the hydrothermal effect on the hemipelagic sediments in the Sumisu Rift.

\section{Authigenic Components in Chemical Composition}

The $\mathrm{MnO}$ contents are influenced by hydrothermal activity and sedimentary environments. In both the forearc and backarc sites of 
Table 4. Contents of rare-earth elements of sediment samples of Sites 791 and 792.

\begin{tabular}{|c|c|c|c|c|c|c|}
\hline $\begin{array}{l}\text { Core, Section, } \\
\text { Interval }(\mathrm{cm})\end{array}$ & $\begin{array}{c}126-791 \mathrm{~A}-4 \mathrm{H}-4 \\
130-150 \mathrm{~cm}\end{array}$ & $\begin{array}{c}126-791 \mathrm{~A}-22 \mathrm{H}-6 \\
45-65 \mathrm{~cm}\end{array}$ & $\begin{array}{c}126-791 \mathrm{~A}-39 \mathrm{X}-1 \\
23-25 \mathrm{~cm}\end{array}$ & $\begin{array}{c}126-791 \mathrm{~B}-13 \mathrm{R}-1 \\
97-99 \mathrm{~cm}\end{array}$ & $\begin{array}{c}126-791 \mathrm{~B}-23 \mathrm{R}-4 \\
6-7 \mathrm{~cm}\end{array}$ & $\begin{array}{c}126-791 \mathrm{~B}-34 \mathrm{R}-2 \\
139-141 \mathrm{~cm}\end{array}$ \\
\hline La & 15.13 & 10.12 & 15.05 & 13.66 & 11.51 & 11.14 \\
\hline $\mathrm{Ce}$ & 31.72 & 23.25 & 33.39 & 27.30 & 22.36 & 23.99 \\
\hline Pr & 3.81 & 3.11 & 4.11 & 3.53 & 2.76 & 3.21 \\
\hline $\mathrm{Nd}$ & 14.62 & 13.45 & 16.64 & 13.36 & 10.32 & 12.82 \\
\hline $\mathrm{Sm}$ & 3.21 & 3.34 & 3.71 & 2.74 & 2.12 & 3.10 \\
\hline $\mathrm{Eu}$ & 0.93 & 1.13 & 1.09 & 1.00 & 0.75 & 1.02 \\
\hline Gd & 3.52 & 4.27 & 4.34 & 3.92 & 2.72 & 3.93 \\
\hline $\mathrm{Tb}$ & 0.62 & 0.69 & 0.67 & 0.64 & 0.47 & 0.73 \\
\hline Dy & 3.43 & 4.30 & 3.98 & 3.15 & 2.75 & 4.39 \\
\hline Ho & 0.78 & 1.06 & 0.91 & 0.84 & 0.62 & 1.09 \\
\hline Er & 2.04 & 2.85 & 2.32 & 2.00 & 1.46 & 2.79 \\
\hline $\mathrm{Tm}$ & 0.47 & 0.48 & 0.42 & 0.50 & 0.38 & 0.60 \\
\hline $\mathrm{Yb}$ & 2.08 & 2.54 & 2.56 & 2.21 & 1.35 & 2.90 \\
\hline Lu & 0.40 & 0.49 & 0.43 & 0.42 & 0.32 & 0.55 \\
\hline
\end{tabular}

Leg 126, we cannot find a large anomaly in the manganese oxide content, which would suggest a hydrothermal origin, as mentioned above. However, the vertical distribution of manganese oxide contents in the core sequences coincides with the lithologic change in the forearc sites (Figs. 2-4). Higher manganese contents were observed in Unit IV of Site 793 and in Unit III of Site 792, which have distinct, small sedimentation rates in the Leg 126 site sequences. Matsumoto et al. (1985) discussed the relationship between manganese contents and sedimentation rates of continental margin sediments and abyssal basin sediments. They concluded that an inverse relationship between manganese contents and sedimentation rates is clear and that this relation can be explained by the dilution effects of terrigenous materials on hydrogenous manganese oxides with a constant rate of

Table 5. Sedimentation rates of lithologic units, Leg 126.

\begin{tabular}{|c|c|c|c|c|}
\hline Site & Unit & $\begin{array}{l}\text { Depth } \\
\text { (mbsf) }\end{array}$ & $\begin{array}{l}\text { Apparent } \\
\text { sedimentation } \\
\text { rate (m/m.y.) }\end{array}$ & $\begin{array}{l}\text { Sedimentaion } \\
\text { rate of muddy } \\
\text { sediments ( } \mathrm{m} / \mathrm{m} . \mathrm{y} .)\end{array}$ \\
\hline 787 & $\begin{array}{l}\text { I } \\
\text { II } \\
\text { III } \\
\text { IVA } \\
\text { IVB }\end{array}$ & $\begin{aligned} 0 & =21.4 \\
21.4 & =40.3 \\
40.3 & =118.9 \\
118.9 & =281.7 \\
281.7 & =320.1\end{aligned}$ & $\begin{array}{c}67 \\
9 \\
26,120 \\
120\end{array}$ & \\
\hline 788 & $\begin{array}{l}\text { IA } \\
\text { IB } \\
\text { IIA } \\
\text { IIB }\end{array}$ & $\begin{aligned} 0 & -229.2 \\
229.2 & -249 \\
249 & =278.6 \\
278.6 & -374\end{aligned}$ & $\begin{array}{c}115 \\
145-230 \\
39-282 \\
282,73,192\end{array}$ & \\
\hline 790 & $\begin{array}{l}\text { I } \\
\text { II }\end{array}$ & $\begin{array}{c}0-165 \\
165=266.6\end{array}$ & $\begin{array}{c}\text { ca. } 1000 \\
90\end{array}$ & $\begin{array}{l}150,5^{\circ} \\
60^{\circ}\end{array}$ \\
\hline 791 & I & $\begin{aligned} 0 & =428.4 \\
428.4 & =834\end{aligned}$ & $\begin{array}{c}\text { ca. } 2200 \\
344\end{array}$ & \\
\hline 792 & $\begin{array}{c}\text { I } \\
\text { II } \\
\text { III } \\
\text { IV }\end{array}$ & $\begin{array}{r}0-183.7 \\
183.7-357.4 \\
357.4=429.3 \\
429.3-783.4\end{array}$ & $\begin{array}{c}81,120,122 \\
43,23 \\
14,4 \\
30, \text { ca. } 300\end{array}$ & $\begin{array}{c}56^{\circ} \\
14 \\
28+\end{array}$ \\
\hline 793 & $\begin{array}{l}\text { IA } \\
\text { IB } \\
\text { III } \\
\text { IV } \\
\text { V }\end{array}$ & $\begin{aligned} 0 & =32.5 \\
32.5 & =98.9 \\
98.9 & =735.7 \\
735.7 & =759.0 \\
759.0 & =1373.1\end{aligned}$ & $\begin{array}{c}105 \\
70 \\
7 \\
80 \text {, ca. } 250\end{array}$ & $\begin{array}{c}7 \\
28 t, 43 t\end{array}$ \\
\hline
\end{tabular}

Notes: ${ }^{*}=$ based on visual descriptions, and $\dagger=$ based on the FMS columns (Hiscott et al., this volume). supply. We recalculated the sedimentation rates of muddy sediments (hemipelagic and pelagic sediments) in both forearc and backarc sites, excluding ash layers, debris-flow deposits, and turbidites (Table 5), based on original onboard visual core descriptions (Taylor, Fujioka, et al., 1990) and FMS columns (Hiscott et al., this volume). The recalculated sedimentation rates and the manganese oxide contents show an inverse relationship (Fig. 12), like that found by Matsumoto et al. (1985) and Sugisaki (1984).

On the REE patterns of the sediments (Figs. 9 and 10), a few samples have a weak negative Ce anomaly, which can be attributed to reduction in the seawater or sediment column (De Baar et al., 1985; Tanaka et al., 1990). Moreover, all samples have a zigzag feature in Ho-Er-Tm-Yb-Lu with variable degrees of curvature. Masuda and Ikeuchi (1979) and Tanaka et al. (1990) recognized a zigzag feature in the heavy REE patterns of seawater, and they interpreted this feature to be a reflection of a partially cut-off lanthanide tetrad effect. Although seawater would have an affect, in part, on the abundances of heavy REEs in the sediments, these zizag patterns are possibly a result of analytical uncertainties because of the very low abundances of $\mathrm{Ho}, \mathrm{Tm}$, and $\mathrm{Lu}$ in the sediments.

\section{Sedimentary Environments and Diagenesis}

The $\mathrm{SiO}_{2}$ contents of the Pliocene-Pleistocene sequence in the backarc sites are higher than those in the forearc sites with corresponding age (Fig. 7). Higher $\mathrm{SiO}_{2}$ contents and higher ratios of $\mathrm{SiO}_{2}$ to $\mathrm{Al}_{2} \mathrm{O}_{3}$ can possibly be attributed to biogenic silica (i.e., to radiolarians and diatoms) and to acidic volcanogenic materials. Because smear slide observations suggest that siliceous biogenic contributions to the chemical composition are negligible, as mentioned already, we conclude that finer grained acidic volcanic materials affected the $\mathrm{SiO}_{2}$ content of the sediments. Quaternary acidic volcanic materials derived from the arc volcanoes have contents of $72 \%-80 \% \mathrm{SiO}_{2}$ and $11 \%-14 \% \mathrm{Al}_{2} \mathrm{O}_{3}$ (Nishimura et al., this volume; Fujioka et al., this volume). The rift basin is surrounded by the rift flanks, and suspended particles are trapped in the basin. On the other hand, the forearc basin is an open basin and almost all of the suspended particles are blown away from the basin. The differences between the sedimentary environments of forearc and backarc basins are suggested by the sediment sequences of thick pumice deposits. The thick pumice deposits of the backarc sites have thick, finer grained (silt- to clay-size) ash in the 
Table 4 (continued).

\begin{tabular}{|c|c|c|c|c|c|c|}
\hline 126-791B-43R-1 & $126-792 \mathrm{~A}-4 \mathrm{H}-6$ & $126-792 B-9 x-2$ & 126-792E-19R-2 & 126-792E-37R-2 & 126-792E-52R-1 & $126-792 \mathrm{E}-66 \mathrm{R}-3$ \\
\hline $31-32 \mathrm{~cm}$ & $100-102 \mathrm{~cm}$ & $13-15 \mathrm{~cm}$ & $120-122 \mathrm{~cm}$ & $62-63 \mathrm{~cm}$ & $94-96 \mathrm{~cm}$ & $105-107 \mathrm{~cm}$ \\
\hline 32.35 & 11.86 & 16.09 & 8.91 & 19.77 & 16.83 & 8.91 \\
\hline 3.97 & 1.66 & 2.32 & 1.46 & 2.78 & 2.62 & 1.49 \\
\hline 14.79 & 6.80 & 9.28 & 5.87 & 12.35 & 11.47 & 6.16 \\
\hline 3.68 & 2.55 & 3.48 & 2.83 & 3.98 & 3.88 & 2.50 \\
\hline 0.60 & 0.51 & 0.63 & 0.54 & 0.76 & 0.67 & 0.49 \\
\hline 3.28 & 2.52 & 3.42 & 2.80 & 4.06 & 3.60 & 2.46 \\
\hline 0.80 & 0.71 & 0.90 & 0.79 & 1.07 & 0.96 & 0.72 \\
\hline 2.06 & 1.70 & 2.23 & 1.88 & 2.62 & 2.62 & 1.69 \\
\hline 0.37 & 0.43 & 0.56 & 0.50 & 0.59 & 0.54 & 0.48 \\
\hline
\end{tabular}

upper part of the sequences, which were trapped in the basin. The forearc sites, however, have no finer grained ash in the upper part of the sequences (Nishimura et al., this volume).

The minimum $\mathrm{CaO}$ contents of the Site 792 and 793 sequences (Figs. 3 and 4) coincide with the turning point of $\mathrm{CaCl}_{2}$ in the pore water (Egeberg et al., 1990). The origin of the unusually high $\mathrm{CaCl}_{2}$ content in the lower part of the Site 792 and 793 sequences is thought to be related to the alteration of volcanogenic materials, which reflects the chemical composition of sediments with a low $\mathrm{CaO}$ content. The sedimentary samples of this area throughout the sequences have generally high contents of $\mathrm{CaO}$, suggesting that they would be highly affected by the high $\mathrm{CaO}$ contents in the volcanic rocks as a sediment source. Moreover, the presence of gypsum-filled fractures in the sequences (Taylor, Fujioka, et al., 1990) implies the possibility of CaO enrichment from secondary minerals formed through a diagenetic alteration.

\section{CONCLUSIONS}

The chemical composition of hemipelagic and pelagic sediments in the Izu-Bonin Arc shows the following features:

1. The chemical composition of the hemipelagic and pelagic sediments of the sequences of Leg 126 is highly variable. The contents of $\mathrm{SiO}_{2}$ and $\mathrm{MgO}$ are very high in several samples, and those of $\mathrm{Cu}$, $\mathrm{Co}$, and $\mathrm{Ni}$ are constantly very low, compared with those of sediments from ocean basins and the Philippine Sea.

2. The major chemical compositions of hemipelagites are highly affected by that of the source land area. As for the Izu-Bonin region, the source has been the Izu-Bonin Arc itself. The change in nature of the arc is indicated by the vertical change of $\mathrm{MgO}$ content of sediments in the forearc sites. The Oligocene sequences with high $\mathrm{MgO}$ contents reflect the effect of the Paleogene immature arc volcanic rocks. Pliocene to Pleistocene sediments have low $\mathrm{MgO}$ contents similar to acidic volcanic rock in the present arc. The REE analyses also demonstrate the large influence of volcanic rocks to the chemical composition of the sediment.

3. No influence of hydrothermal activity on the chemical features of hemipelagites can be found in the core sequences of the Sumisu Rift, although high $\mathrm{MnO}$ contents in the surface sediments and hydrothermal vents were observed in the rift.

4. The $\mathrm{MnO}$ values are not so large throughout the sequences, but the values do show an inverse correlation with sedimentation rates.
The highest values are recorded in the chalk with a very small sedimentation rate deposited in the pelagic environment.

5. A contrast in chemical composition between the forearc and backarc sites is suggested by the $\mathrm{SiO}_{2}$ content. The values of $\mathrm{SiO}_{2}$ in the backarc are higher than in the forearc, which is probably related to the topography of the rift basin, which is surrounded by rift flanks that trap suspended finer grained acidic volcanogenic materials.

\section{ACKNOWLEDGMENTS}

We thank Dr. R. Hiscott for permission to use FMS columns to recalculate the sedimentation rates and references of sandstone geochemistry in this area. We would like to thank S. Nakao, M. Yuasa, K. Rodolfo, K. Marsaglia, and K. Fujioka for extensive and fruitful discussions regarding the sedimentation and geologic history of the Izu-Bonin Arc. We are very grateful to R. Sugisaki and T. Plank for reviewing the manuscript.

\section{REFERENCES}

Brown, G., and Taylor, B., 1988. Sea-floor mapping of the Sumisu Rift, Izu-Ogasawara (Bonin) Island Arc. Bull. Geol. Surv. Jpn., 39:23-38.

Burke, W. H., Denison, R. E., Hetherington, E. A., Koepnic, R. B., Nelson, H. F., and Otto, J. B., 1982. Variation of seawater ${ }^{87} \mathrm{Sr} /{ }^{86} \mathrm{Sr}$ throughout Phanerozoic time. Geology, 10:516-519.

De Baar, H.J.W., Bacon, M. P., Brewer, P. G., and Bruland, K. W., 1985. Rare earth elements in the Pacific and Atlantic Oceans. Geochim. Cosmochim. Acta, 49:1943-1959.

Egeberg, P. K., and Leg 126 Shipboard Scientific Party, 1990. Unusual composition of pore waters found in the Izu-Bonin fore-arc sedimentary basin. Nature, 344:215-218.

Fryer, P., Taylor, B., Langmuir, C. H., and Hochstaedter, A. G., 1990. Petrology and geochemistry of lavas from the Sumisu and Torishima backarc rifts. Earth Planet. Sci. Lett., 100:161-178.

Hess, J., Bender, M. L., and Schilling, J.-G., 1986. Evolution of the ratio of strontium- 87 to strontium- 86 in seawater from Cretaceous to Present. Science, 231:979-984.

Hickey, R. L., and Frey, F. A., 1982. Geochemical characteristics of boninite series volcanism: implications for their source. Geochim. Cosmochim. Acta, 46:2099-2115.

Hochstaedter, A. G., Gill, J. B., and Morris, J. D., 1990. Volcanism in the Sumisu Rift, II. Subduction and non-subduction related components. Earth Planet. Sci. Lett., 100:195-209.

Honza, E., and Tamaki, K., 1985. The Bonin Arc. In Nairn, A.E.M., Stehli, F. G., and Uyeda, S. (Eds.), The Ocean Basins and Margins (Vol. 7): The Pacific Ocean: New York (Plenum Press), 459-499. 

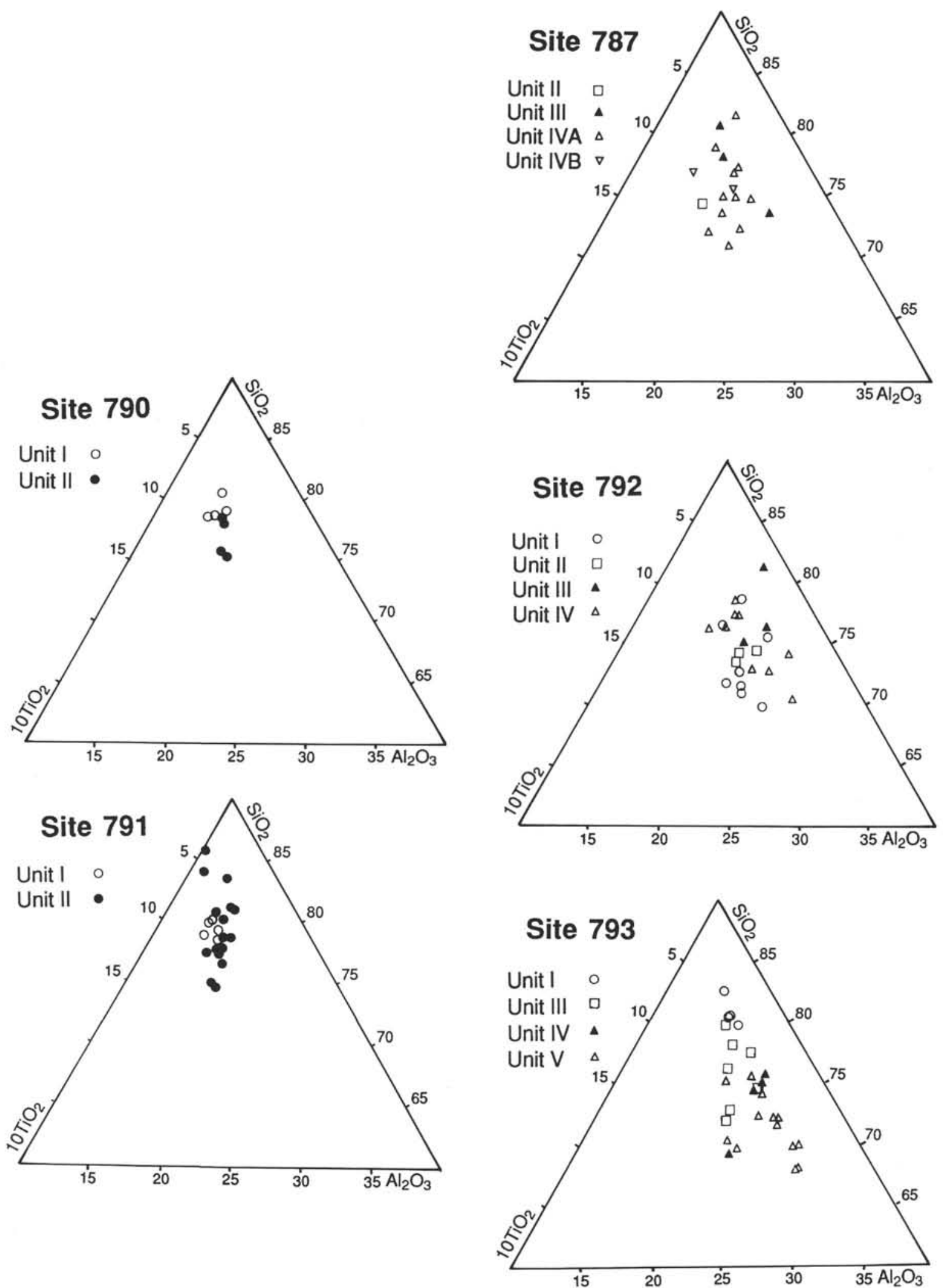

Figure 7. $\mathrm{SiO}_{2}-\mathrm{Al}_{2} \mathrm{O}_{3}-10 \mathrm{TiO}_{2}$ triangles of $\mathrm{Leg} 126$ samples. 

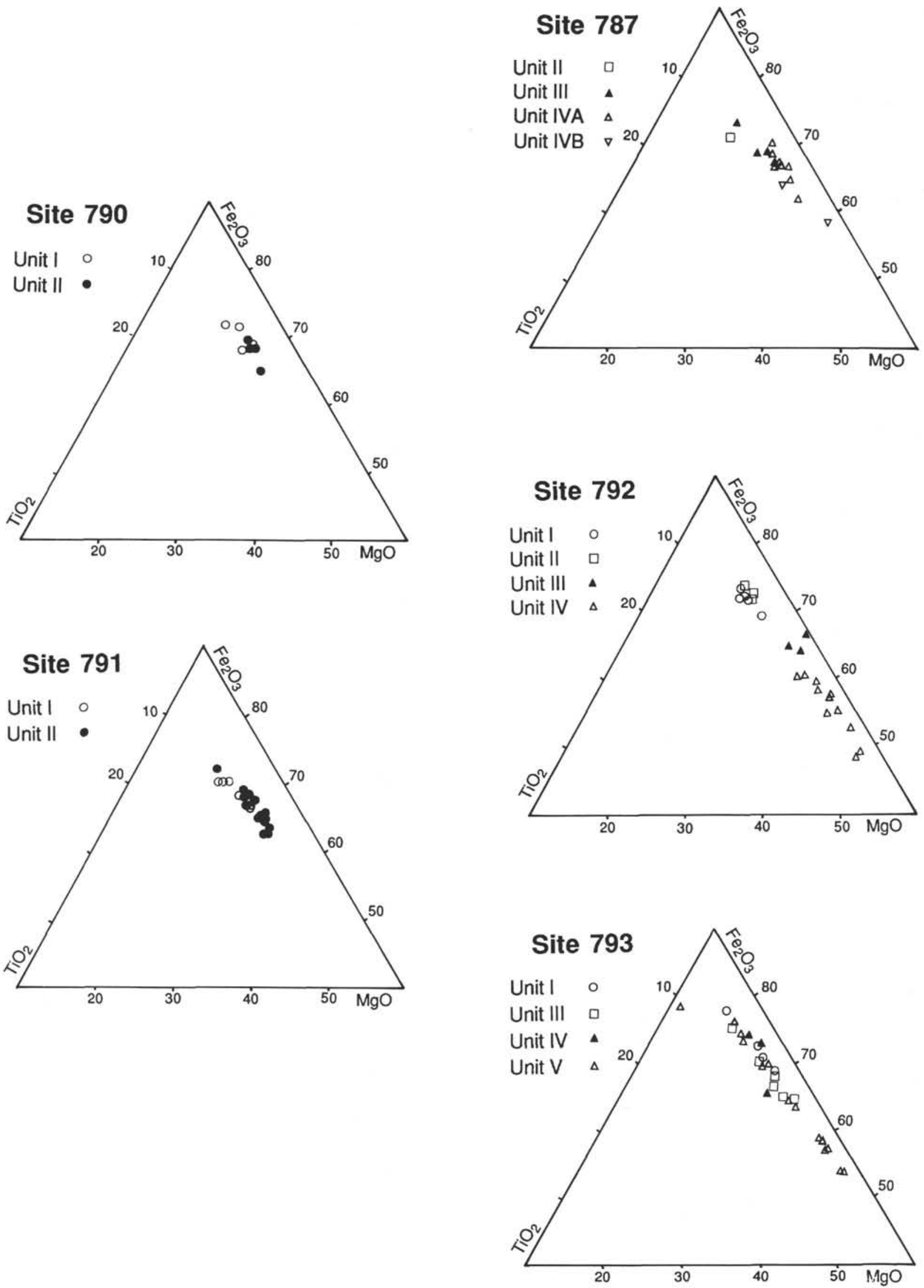

Figure 8. $\mathrm{Fe}_{2} \mathrm{O}_{3}-\mathrm{MgO}-\mathrm{TiO}_{2}$ triangles of Leg 126 samples. 


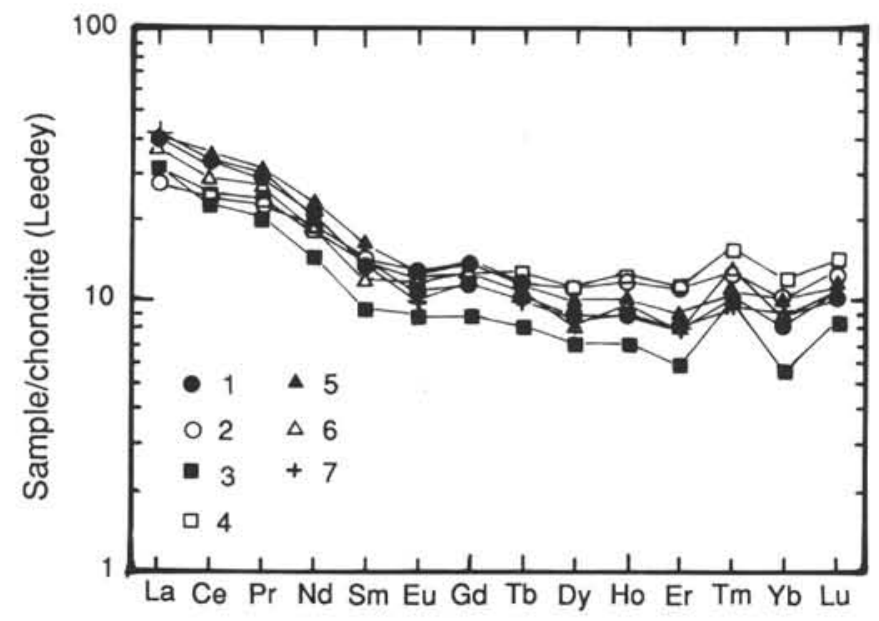

Figure 9. Chondrite-normalized REE patterns for sediment samples at Site 791. $1=$ Sample $126-791 \mathrm{~A}-4 \mathrm{H}-4,130-150 \mathrm{~cm} ; 2=$ Sample 126-791A-22H-6, $45-65 \mathrm{~cm}$; $3=$ Sample 126-791A-39X-1, 23-25 cm; 4 = Sample 126-791B-13R-1, $97-99 \mathrm{~cm}$; $5=$ Sample 126-791B-23R-4, 6-7 cm; 6 = Sample 126-791B-34R-2, 139-141 cm; and $7=$ Sample 126-791B-43R-1, 31-32 cm.

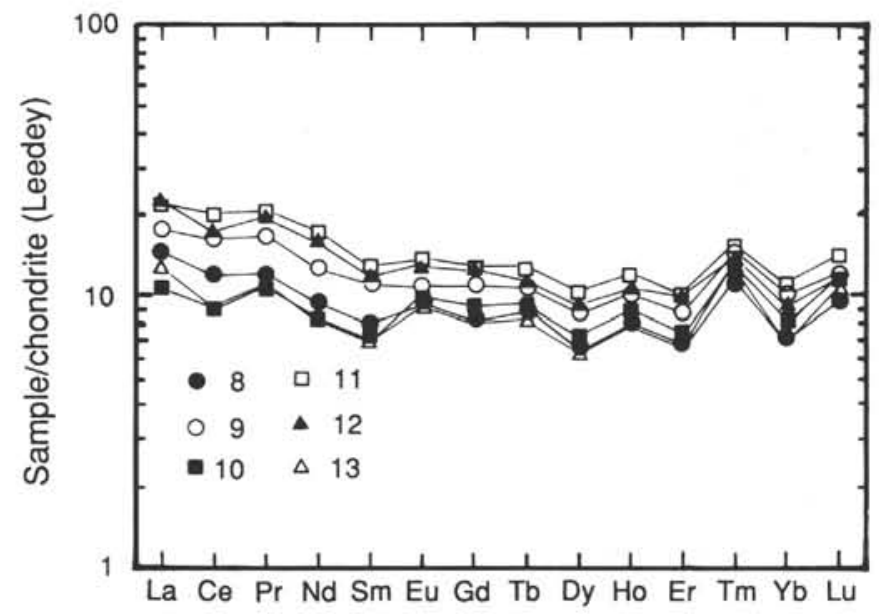

Figure 10. Chondrite-normalized REE patterns for sediment samples at Site 792. $8=$ Sample 126-792A-4H-6, 100-102 cm; 9 = Sample 126-792B-9X-2, 13-15 cm; $10=$ Sample 126-792E-19R-2, 120-122 cm; 11 = Sample 126-792E-37R-2, $62-63 \mathrm{~cm} ; 12$ = Sample 126-792E-52R-1, 94-96 cm; and $13=$ Sample $126-$ 792E-66R-3, $105-107 \mathrm{~cm}$.

Ikeda, Y., and Yuasa, M., 1989. Volcanism in nascent back-arc basins behind the Shichito Ridge and adjacent areas in the Izu-Ogasawara arc, northwest Pacific: evidence for mixing between E-type MORB and island arc magmas at the initiation of back-arc rifting. Contrib. Mineral. Petrol., 101:377-393.

Kuroda, N., and Shiraki, K., 1975. Boninite and related rocks of Chichijima, Bonin Islands, Japan. Rep. Fac. Sci., Shizuoka Univ., 10:145-155.

Leinen, M., 1987. The origin of paleochemical signatures in North Pacific pelagic clays: partitioning experiments. Geochim. Cosmochim. Acta, 51:305-319.

Masuda, A., and Ikeuchi, Y., 1979. Lanthanide tetrad effect observed in marine environment. Geochem. J., 13:19-22.

Matsumoto, R., Minai, Y., and lijima, A., 1985. Manganese content, cerium anomaly, and rate of sedimentation as aids in the characterization and classification of deep-sea sediments. In Nasu, N., Kobayashi, K., Uyeda, S., Kushiro, I., and Kagami, H. (Eds.) Formation of Active Ocean Margins: Tokyo (Terra Scientific), 913-939.

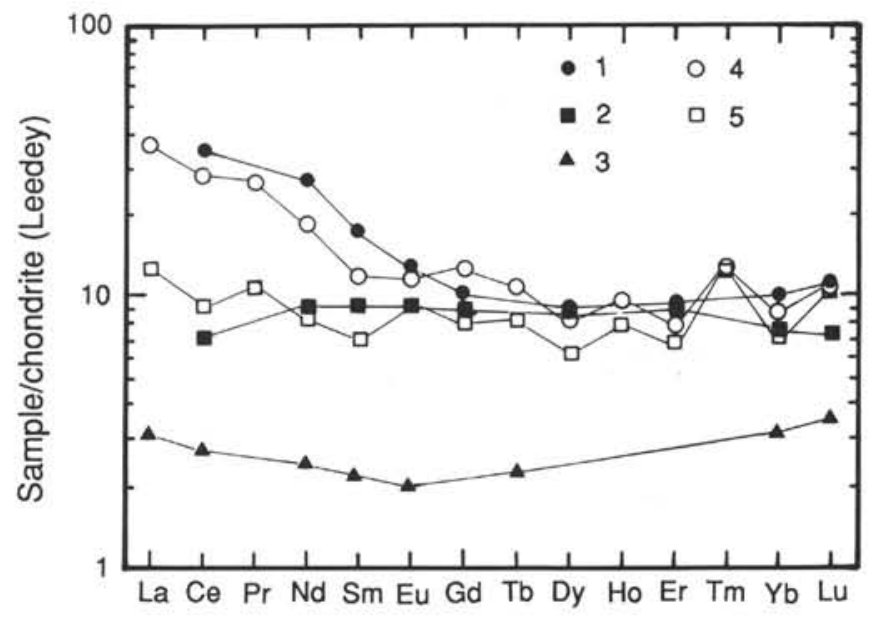

Figure 11. Chondrite-normalized REE patterns for selected sediment samples compared with volcanic rocks of the Izu-Bonin Arc. 1 = dacite from the backarc seamount (Sample D623-2; Ikeda and Yuasa, 1989); 2 = basalt from the arc ridge (Sample D640-1; Ikeda and Yuasa, 1989); 3 = boninitic rock from Bonin Island (Sample 2983; Hickey and Frey, 1982); 4 = backarc sediment sample (Sample 126-791B-34R-2, 139-141 cm; this study); and 5 = forearc sediment sample (Sample 126-792E-66R-3, 105-107 cm; this study).

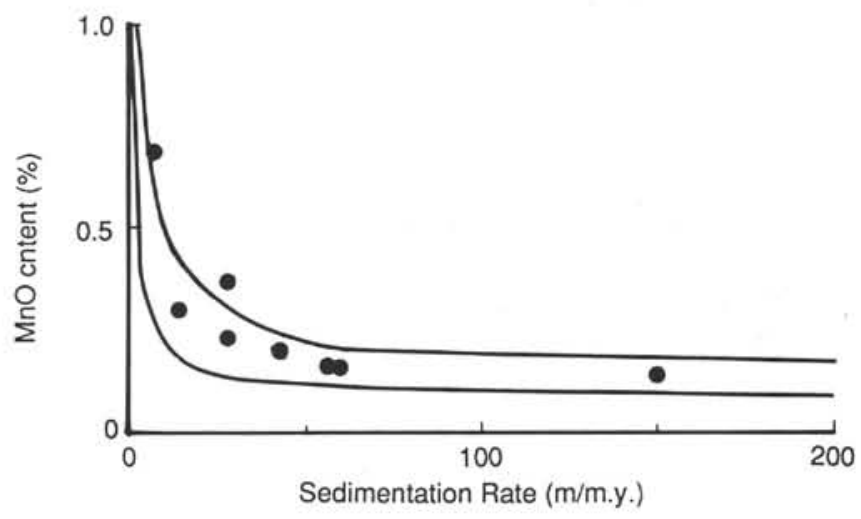

Figure 12. Relationship between $\mathrm{MnO}$ content and sedimentation rate. The area between two solid lines shows modern sediments in Matsumoto et al. (1985).

Mita, N., Nakao, S., and Kato, K., 1982. Minor chemical composition of bottom sediments from the Central Pacific Wake-Tahiti Transect. Cruise Rep., Geol. Surv. Jpn., 18:313-337.

Murakami, F., 1988. Structural framework of the Sumisu Rift, Izu-Ogasawara Arc. Bull. Geol. Surv. Jpn., 39:1-21.

Nakao, S., Yuasa, M., Nohara, M., and Usui, A., 1990. Submarine hydrothermal activity in the Izu-Ogasawara Arc, western Pacific. Rev. Aquatic Sci., 3:95-115.

Nishimura, A., and Murakami, F., 1988. Sedimentation in the Sumisu Rift, Izu-Ogasawara Arc. Bull. Geol. Surv. Jpn., 39:39-61.

Nishimura, A., Yamazaki, T., Yuasa, M., Mita, N., and Nakao, S., 1988. Bottom sample and heat flow data of Sumisu and Torishima Rifts, Izu-Ogasawara Island Arc. Geol. Surv. Jpn., Mar. Geol. Map Ser., 31 (scale 1:200,000).

Nohara, M., 1980. Geochemical history of Japan Trench sediments sampled during Leg 56, Deep Sea Drilling Project. In Langseth, M., Okada, H., et al., Init. Repts. DSDP, 56, 57, Pt. 2: Washington (U.S. Govt. Printing Office), 1251-1257.

Nohara, M., and Kato, K., 1985. Chemical compositions of pelagic deep-sea sediments-its relation to the formation of authigenic mineral phase under the chemical control of sea water. In Nasu, N., Kobayashi, K., Uyeda, S., Kushiro, I., and Kagami, H. (Eds.), Formation of Active Ocean Margins: Tokyo (Terra Scientific), 893-912. 
Peterman, Z. E., Hedge, C. E., and Tourtelot, A., 1970. Isotopic composition of strontium in seawater throughout Phanerozoic. Geochim. Cosmochim. Acta, 34:105-120.

Shipboard Scientific Party, 1990a. Correlation and comparison of forearc sites. In Taylor, B., Fujioka, K., et al., Proc. ODP. Init. Repts., 126: College Station, TX (Ocean Drilling Program), 407-413.

, 1990b. Correlation and comparison of backarc sites. In Taylor, B. Fujioka, K., et al., Proc. ODP, Init. Repts., 126: College Station, TX (Ocean Drilling Program), 415-418.

Sugisaki, R., 1978. Chemical composition of argillaceous sediments on the Pacific margins of southwest Japan. Cruise Rep., Geol. Surv. Jpn., 9:65-73.

1980a. Major element chemistry of the Japan Trench sediments, Legs 56 and 57, Deep Sea Drilling Project. In Langseth, M., Okada, H., et al., Init. Repts. DSDP, 56, 57, Pt. 2: Washington (U.S. Govt. Printing Office), 1233-1249.

1980b. Major-element chemistry of argillaceous sediments at Deep Sea Drilling Project Sites 442, 443, and 444, Shikoku Basin. In Klein, G. deV., Kobayashi, K., et al., Init. Repts. DSDP, 58: Washington (U.S. Govt. Printing Office), 719-735.

, 1984. Relation between chemical composition and sedimentation rate of Pacific ocean-floor sediments deposited since the middle Cretaceous: basic evidence for chemical constraints on depositional environments of ancient sediments. J. Geol., 92:235-259.

Sugisaki, R., and Kinoshita, T., 1981. Chemical composition of marine argillaceous sediments around the Izu-Ogasawara islands. Cruise Rep., Geol. Surv. Jpn., 14:146-158.
Tanaka, M., Shimizu, H., and Masuda, A., 1990. Features of the heavy rare-earth elements in seawater. Geochem. J., 24:39-46.

Taylor, B., Brown, G., Fryer, P., Gill, J., Hochstaedter, A., Hotta, H., Langmuir, C., Leinen, M., Nishimura, A., and Urabe, T., 1990. ALVIN-SeaBeam studies of the Sumisu Rift, Izu-Bonin Arc. Earth Planet. Sci. Lett., 100:127-147.

Taylor, B., Fujioka, K., et al., 1990. Proc. ODP, Init. Repts., 126: College Station, TX (Ocean Drilling Program).

Umino, S., 1985. Volcanic geology of Chichijima, the Bonin Islands (Ogasawara Islands). J. Geol. Soc. Jpn., 91:505-523.

Urabe, T., and Kusakabe, M., 1990. Barite silica chimneys from the Sumisu Rift, Izu-Bonin Arc: possible analog to hematitic chert associated with Kuroko deposits. Earth Planet. Sci. Lett., 100:283-290.

Usui, A., Yuasa, M., Nohara, M., and Murakami, F., 1986. Submarine hydrothermal manganese deposits from the Ogasawara (Bonin) Arc. Mar. Geol., 73:311-322.

Yamazaki, T., 1988. Heat flow in the Sumisu Rift, Izu-Ogasawara (Bonin) Arc. Bull. Geol. Surv. Jpn., 39:63-70.

Date of initial receipt: 2 January 1991

Date of acceptance: 19 August 1991

Ms 126B-151 